\title{
On hyperplane sections of K3 surfaces
}

\author{
Enrico Arbarello, Andrea Bruno and Edoardo Sernesi
}

\begin{abstract}
Let $C$ be a Brill-Noether-Petri curve of genus $g \geqslant 12$. We prove that $C$ lies on a polarised K3 surface, or on a limit thereof, if and only if the Gauss-Wahl map for $C$ is not surjective. The proof is obtained by studying the validity of two conjectures by J. Wahl. Let $\mathcal{I}_{C}$ be the ideal sheaf of a non-hyperelliptic, genus $g$, canonical curve. The first conjecture states that if $g \geqslant 8$ and if the Clifford index of $C$ is greater than 2, then $H^{1}\left(\mathbb{P}^{g-1}, \mathcal{I}_{C}^{2}(k)\right)=0$ for $k \geqslant 3$. We prove this conjecture for $g \geqslant 11$. The second conjecture states that a Brill-Noether-Petri curve of genus $g \geqslant 12$ is extendable if and only if $C$ lies on a K3 surface. As observed in the introduction, the correct version of this conjecture should admit limits of polarised K3 surfaces in its statement. This is what we prove in the present work.
\end{abstract}

\section{Introduction}

The fundamental result of Brill-Noether theory for curves, as proved by Griffiths and Harris in [GH80], states that, on a general curve of genus $g$, there is no $g_{d}^{r}$ as soon as the Brill-Noether number

$$
\rho(g, r, d)=g-(r+1)(g-d+r)
$$

is negative. As usual, by a $g_{d}^{r}$ we mean a linear series associated with an $(r+1)$-dimensional subspace $V$ of the space of global sections of a line bundle $L$ of degree $d$ on $C$. Associated with $L$ is the so-called Petri map

$$
\mu_{0, L}: H^{0}(C, L) \otimes H^{0}\left(K L^{-1}\right) \longrightarrow H^{0}(C, K),
$$

where $K=K_{C}$ is the canonical bundle on $C$. The Brill-Noether number is nothing but the difference between the dimensions of the codomain and the domain of this map. A stronger version of the Brill-Noether theorem, as proved by Gieseker in [Gie82], states that on a general curve of genus $g$, the Petri map $\mu_{0, L}$ is injective for every line bundle $L$ on $C$. A curve for which the Petri map $\mu_{0, L}$ is injective for every line bundle $L$ on it is said to be a Brill-Noether-Petri curve or, for short, a BNP curve (cf. [ACGH85, ACG11]). A breakthrough in Brill-Noether theory came when Lazarsfeld [Laz86] proved Gieseker's theorem by showing that a genus $g$ curve $C$ lying on a K3 surface $S$ having the property that $\operatorname{Pic}(S)=\mathbb{Z} \cdot C$ is a $\mathrm{BNP}$ curve.

Received 8 November 2016, accepted in final form 10 November 2016.

2010 Mathematics Subject Classification 14J10, 14J28, 14 H51.

Keywords: K3 surfaces, Brill-Noether-Petri curves, Gauss-Wahl map.

This journal is (C) Foundation Compositio Mathematica 2017. This article is distributed with Open Access under the terms of the Creative Commons Attribution Non-Commercial License, which permits non-commercial reuse, distribution, and reproduction in any medium, provided that the original work is properly cited. For commercial re-use, please contact the Foundation Compositio Mathematica.

The first-named author would like to thank the Institute of Advanced Study for hospitality during the preparation of part of this work. The second- and third-named authors were partly supported by the project MIUR-PRIN 2010/11 "Geometria delle Varietà Algebriche". 


\section{ON HYPERPLANE SECTIONS OF K3 SURFACES}

Lazarsfeld's theorem also shows that it is not possible to characterise the locus of curves lying on K3 surfaces by looking at line bundles on them. One may look at vector bundles, and then the situation is completely different. In fact, following an idea of Mukai, the authors of this paper where able to describe a general K3 surface in terms of an exceptional Brill-Noether locus for vector bundles on one of its hyperplane sections [ABS14]. Here we take a different point of view.

The starting point is a theorem by Wahl [Wah87] proving that for a smooth curve $C$ of genus $g \geqslant 2$ lying on a K3 surface $S$, the Gaussian map (also called the Gauss-Wahl map or Wahl map)

$$
\begin{aligned}
\nu: \wedge^{2} H^{0}(C, K) & \longrightarrow H^{0}(C, 3 K) \\
s \wedge t & \longmapsto s \cdot d t-t \cdot d s
\end{aligned}
$$

is not surjective. A beautiful geometric proof of this fact was given by Beauville and Merindol [BM87]. This theorem is all the more significant in light of the fact that for a general curve, the Wahl map is surjective. This was proved, with different methods, by Ciliberto, Harris, Miranda [CHM88], and by Voisin [Voi92]. In this last paper Voisin, for the first time, suggests to study the non-surjectivity of the Wahl map under the Brill-Noether-Petri condition, in order to characterise hyperplane sections of K3 surfaces (see [Voi92, Remark 4.13(b)], where the author also refers to Mukai). In [CU93], Cukierman and Ulmer proved that when $g=10$, the closure of the locus in $M_{10}$ of curves that are canonical sections of K3 surfaces coincides with the closure of the locus of curves with non-surjective Wahl map. This locus has been studied in more detail by Farkas-Popa in [FP05], where they gave a counterexample to the slope conjecture.

Our aim is to prove the following theorem.

Theorem 1.1. Let $C$ be a Brill-Noether-Petri curve of genus $g \geqslant 12$. Then $C$ lies on a polarised K3 surface, or on a limit thereof, if and only if its Wahl map is not surjective.

Our investigation was sparked by a remarkable paper by Wahl [Wah97], where the author analyses the significance of the non-surjectivity of his map from the point of view of deformation theory.

Let $C \subset \mathbb{P}^{g-1}$ be a canonically embedded curve of genus $g \geqslant 3$. Wahl finds a precise connection between the cokernel of $\nu$ and the deformation theory of the affine cone over $C$. Denoting by

$$
A=\oplus \Gamma\left(C, \mathcal{O}_{C}(k)\right)
$$

the coordinate ring of this cone, Wahl considers the graded cotangent module $T_{A}^{1}$ and shows that

$$
\left(T_{A}^{1}\right)_{-1} \cong(\operatorname{Coker} \nu)^{\vee} \text {. }
$$

He also gives a precise interpretation of the graded pieces of the obstruction module $T_{A}^{2}$ :

$$
\left(T_{A}^{2}\right)_{k} \cong H^{1}\left(\mathbb{P}^{g-1}, \mathcal{I}_{C / \mathbb{P}}^{2}(1-k)\right)^{\vee},
$$

where $\mathcal{I}_{C / \mathbb{P}}$ is the ideal sheaf of $C \subset \mathbb{P}^{g-1}$. The modules $T_{A}^{1}$ and $T_{A}^{2}$ govern the deformation theory of the cone $\operatorname{Spec} A$. The canonical curve $C \subset \mathbb{P}^{g-1}$ is said to be extendable if it is a hyperplane section of a surface $\bar{S} \subset \mathbb{P}^{g}$ which is not a cone. The main theorem of [Wah97] is the following.

TheOrem 1.2 (Wahl). Let $C \subset \mathbb{P}^{g-1}$ be a canonical curve of genus $g \geqslant 8$, with $\operatorname{Cliff}(C) \geqslant 3$. Suppose that

$$
H^{1}\left(\mathbb{P}^{g-1}, \mathcal{I}_{C / \mathbb{P}}^{2}(k)\right)=0, \quad k \geqslant 3 .
$$

Then $C$ is extendable if and only if $\nu$ is not surjective. 


\section{E. Arbarello, A. Bruno and E. Sernesi}

Wahl then conjectures that every canonical curve of Clifford index greater than or equal to 3 satisfies the vanishing condition (1.1). We recall that the Clifford index of a curve $C$ is the minimum value of $\operatorname{deg} D-2 \operatorname{dim}|D|$ taken over all linear systems $|D|$ on $C$ such that $\operatorname{dim}|D| \geqslant 1$ and $\operatorname{dim}\left|K_{C}-D\right| \geqslant 1$. With a slight restriction on the genus, this is what we prove in the first part of the present paper.

Theorem 1.3. Let $C \subset \mathbb{P}^{g-1}$ be a canonically embedded curve of $g \geqslant 11$. Suppose $\operatorname{Cliff}(C) \geqslant 3$. Let $\mathcal{I}_{C / \mathbb{P}} \subset \mathcal{O}_{\mathbb{P}^{g-1}}$ be the ideal sheaf of $C$. Then

$$
H^{1}\left(\mathbb{P}^{g-1}, \mathcal{I}_{C / \mathbb{P}}^{2}(k)\right)=0
$$

for all $k \geqslant 3$.

From Theorem 1.2, one gets the following obvious corollary.

Corollary 1.4. Let $C \subset \mathbb{P}^{g-1}$ be a canonical curve of genus $g \geqslant 11$ with $\operatorname{Cliff}(C) \geqslant 3$. Then $C$ is extendable if and only if $\nu$ is not surjective.

To say that $C$ is extendable means that there exists a projective surface $\bar{S} \subset \mathbb{P}^{g}$, not a cone, having $C$ as a hyperplane section. In general, the surface $\bar{S}$ has isolated singularities, and it is only when there is a smoothing of $\bar{S}$ in $\mathbb{P}^{g}$ that we can say that $\bar{S}$ is the limit of a K3 surface.

In his paper, Wahl gives a nice example of an extendable curve $C \subset \bar{S} \subset \mathbb{P}^{g}$ for which $\bar{S}$ is non-smoothable. In the example, the curve $C$ has a realisation as a smooth plane curve of degree $d \geqslant 7$ and therefore is highly non-BNP. This is perhaps one of the reasons that lead Wahl to conjecture that " $A$ Brill-Noether-Petri curve of genus $g \geqslant 8$ sits on a K3 surface if and only if the Gaussian is not surjective". This conjecture, as stated, is slightly incorrect. Indeed, in the two recent papers [ABFS16] and [AB17], it is shown that there are plane curves (the du Val curves) that are BNP curves, with non-surjective Gauss-Wahl map (for every value of the genus), which cannot be realised as hyperplane sections of a smooth K3 surface (for every odd value of the genus greater than 12). Thus, from this point of view, the statement of Theorem 1.1 is the correct one.

We now proceed to give a brief presentation of Parts I and II of our paper.

Part I. One of the results in [Wah97] is that, indeed, the vanishing condition (1.1) holds for a general curve of genus $g \geqslant 8$. The way Wahl proves this vanishing theorem is by showing that this is true for a class of pentagonal curves of genus $g \geqslant 8$. Recall that associated with a complete pencil of degree $d$ on $C$, in short, a complete $g_{d}^{1}$, is a scroll $X\left(g_{d}^{1}\right) \subset \mathbb{P}^{g-1}$ containing $C$. The set-theoretic description of $X\left(g_{d}^{1}\right)$ is given by

$$
X\left(g_{d}^{1}\right)=\underset{D \in g_{d}^{1}}{\cup}\langle D\rangle,
$$

where $\langle D\rangle \cong \mathbb{P}^{d-2}$ is the linear span in $\mathbb{P}^{g-1}$ of the divisor $D$. The scroll $X\left(g_{d}^{1}\right)$ is a $(d-1)$ dimensional variety of degree $g-d+1$ whose desingularisation is a projective bundle over $\mathbb{P}^{1}$. For $g \geqslant 8$, it is possible to choose a curve $C$ with a base-point-free $g_{5}^{1}$ such that the scroll $Y=X\left(g_{5}^{1}\right)$ is a smooth 4 -fold. Wahl proves that to check the vanishing statement (1.1) for such a curve $C$, it is enough to verify the analogous statement

$$
H^{1}\left(Y, \mathcal{I}_{C / Y}^{2}(k)\right)=0, \quad k \geqslant 3
$$

where $\mathcal{I}_{C / Y}$ is the defining ideal sheaf of $C$ on $Y$. For the 4 -fold $Y$ it is not difficult to compute a resolution of the ideal sheaf $\mathcal{I}_{C / Y}$, and the vanishing result (1.2) follows easily. 


\section{ON HYPERPLANE SECTIONS OF K3 SURFACES}

Our proof of Theorem 1.3 evolves, broadly, along the same lines of Wahl's proof of the vanishing (1.1) in the pentagonal case. The questions are: what is the right scroll that could serve the same purpose as $X\left(g_{5}^{1}\right)$ ? and how can one use the hypothesis that $\operatorname{Cliff}(C) \geqslant 3$ ? The answer to both questions comes from a remarkable theorem by Voisin, which generalises to the case $\operatorname{Cliff}(C) \geqslant 3$ a theorem by Green and Lazarsfeld regarding curves with $\operatorname{Cliff}(C) \geqslant 2$. This is Theorem 2.3 below, which characterises curves with $\operatorname{Cliff}(C) \geqslant 3$. This characterisation consists of the existence on $C$ of a base-point-free $g_{g-2}^{1}$ whose residual is a base-point-free $g_{g}^{2}$ giving a birational realisation of $C$ in $\mathbb{P}^{2}$ as an irreducible curve of degree $g$ with only double points as singularities. It is then natural to look at the scroll $X=X\left(g_{g-2}^{1}\right) \subset \mathbb{P}^{g-1}$. A slight disadvantage of this scroll is that it is singular; a more serious disadvantage is that it is of high dimension (equal to $g-3$ ). On the positive side, $X$ is of low degree, in fact it is a cubic projecting the Segre variety $\mathbb{P}^{1} \times \mathbb{P}^{2} \subset \mathbb{P}^{5}$, and $C$ lies entirely in the non-singular locus of $X$. As in the pentagonal case, the reduction of the vanishing statement (1.1) to the vanishing statement

$$
H^{1}\left(X, \mathcal{I}_{C / X}^{2}(k)\right)=0, \quad k \geqslant 3
$$

is rather uneventful (see Section 1.3). The heart of the matter is to prove (1.3).

What comes to our aid is the plane representation of $C$ via the $g_{g}^{2}$ of Voisin's theorem (see Section 4). Let $\Gamma \subset \mathbb{P}^{2}$ be the plane curve given by this $g_{g}^{2}$. Via the adjoint linear system to $\Gamma$, the projective plane is mapped birationally to a surface $P \subset \mathbb{P}^{g-1}$ with isolated singularities. This surface is contained in $X$ and contains $C$ in its smooth locus. One then looks at the diagram (6.1) linking the conormal bundle to $C$ in $X$ to the restriction to $C$ of the conormal bundle to $P$ in $X$ (in diagram (6.1) we treat the case $k=3$, but the case $k \geqslant 3$ is analogous). The vanishing of $H^{1}\left(X, \mathcal{I}_{C / X}^{2}(3)\right)$ is equivalent to the surjectivity of the natural homomorphism $H^{0}\left(X, \mathcal{I}_{C / X}(3)\right) \rightarrow H^{0}\left(C, N_{C / X}^{\vee}(3 H)\right)$. Looking at the exact sequence

$$
0 \rightarrow N_{P / X \mid C}^{\vee}(3 H) \rightarrow N_{C / X}^{\vee}(3 H) \rightarrow \mathcal{O}_{C}(3 H-C) \rightarrow 0,
$$

it is not hard to show that this, in turn, is equivalent to the surjectivity of the map $H^{0}\left(X, \mathcal{I}_{P / X}(3)\right)$ $\rightarrow H^{0}\left(C, N_{P / X \mid C}^{\vee}(3 H)\right)$. What comes next is a rather unexpected fact, namely that a suitable twist of the restricted conormal bundle $N_{P / X \mid C}^{\vee}$ is trivial (see Section 5). This makes all the computations straightforward and brings the proof to a quick conclusion (see Section 6).

The cohomology of the square of an ideal sheaf has attracted quite a bit of interest and the reader may consult [BEL91, Ber97, Ver02] for related results.

Part II. In the second part, we start from an extendable canonical curve $C$. This means that there exists a projective surface $\bar{S} \subset \mathbb{P}^{g}$, not a cone, having $C$ as a hyperplane section. In general, the surface $\bar{S}$ has isolated singularities. These surfaces have been completely classified by Epema [Epe83, Epe84] (his work and the works by Ciliberto and Lopez [CL02], du Val [duV33] and Umezu [Ume81], were all important in our study). The classification of these surfaces runs as follows. Consider the maps

$$
p: S \longrightarrow S_{0}, \quad q: S \longrightarrow \bar{S} \subset \mathbb{P}^{g},
$$

where $q$ is the minimal resolution and $S_{0}$ a minimal model. Epema proves (see Proposition 9.2 below) that $\bar{S}$ is projectively normal, that $h^{0}\left(S,-K_{S}\right)=1$ and that $S$ is either a K3 surface or a ruled surface. The smooth surface $S$ is equipped with a polarisation $C$, with $C^{2}=2 g-2$ and $h^{0}(S, C)=g+1$, and with an anti-canonical divisor $Z \sim-K_{S}$ with $C \cdot Z=0$. The 


\section{E. Arbarello, A. Bruno and E. Sernesi}

linear system $|C|$ maps $S$ to $\bar{S}$, and when $Z \neq 0$, it contracts $Z$ to one or two isolated singularities, depending on whether $Z$ possesses one or two connected components. If $S_{0}$ is not a smooth K3 surface, then either $S_{0} \cong \mathbb{P}^{2}$ or $S_{0} \cong \mathbb{P}(E)$, where $E$ is a rank 2 vector bundle over a curve $\Gamma$.

The task at hand becomes clear. In order to prove Theorem 1.1, we must show that if the hyperplane section $C$ of $\bar{S}$ is a Brill-Noether-Petri curve, then either $\bar{S}$ is a (possibly singular) $\mathrm{K} 3$ surface or it is smoothable in $\mathbb{P}^{g}$ as a surface with canonical sections.

A necessary condition for this to happen is that the singularities of $\bar{S}$ are smoothable. A first step consists in showing that, in fact, this is all we need to check. Indeed, a general deformation theory argument, based on the nature of surfaces with canonical sections, shows that once the local smoothability holds, the surface $\bar{S}$ is smoothable in $\mathbb{P}^{g}$ as a surface with canonical sections.

Let us see how the BNP condition reflects itself in the local smoothability of the singularities of $\bar{S}$. An example helps to elucidate the situation. For this we limit ourselves to the case $S_{0} \cong \mathbb{P}^{2}$. It easily follows from the above description of $\bar{S}, S$ and $S_{0}$ that the plane model of $C$ is a curve $C_{0}$ of degree $d$ whose singularities all lie on a cubic $J_{0}$ (which is nothing but the image in $\mathbb{P}^{2}$ of the anti-canonical curve $Z$ ). Let us assume for simplicity that $C_{0}$ has only ordinary multiple points. The surface $S$ is obtained from $S_{0}$ by blowing up all the points of intersection $C_{0} \cap J_{0}$. If $h$ is the cardinality of $C_{0} \cap J_{0}$, then $Z^{2}=9-h$. It is well known that when $Z^{2}$ is very negative, the singularity of $\bar{S}$, obtained by contracting $Z$, is not smoothable. Now, suppose that $C_{0}$ has only double points as singularities, and let $\delta$ be their number. Certainly, when $g$ is big, $h=3 d-\delta$ can be much bigger than 9 , so that the number $Z^{2}$ can be very negative and $\bar{S}$ not smoothable. How is this prevented from a Brill-Noether condition? Simply by Bézout's theorem, telling us that $3 d \geqslant 2 \delta$, so that the genus formula gives $2 g \geqslant d^{2}-6 d+2$, whereas the Brill-Noether requirement for a $g_{d}^{2}$ is that $3 d \geqslant 2 g+6$, leaving us with a meagre $d \leqslant 8$.

By general arguments, one also sees that the elliptic singularity of $\bar{S}$ is smoothable as soon as $Z^{2} \geqslant-9$.

What all of this teaches us is that the BNP condition has a definite effect on the number of singular points of $C_{0}$ and therefore on the number $Z^{2}$. In fact, in our analysis for the case $S_{0} \cong \mathbb{P}^{2}$, we see that as soon as $g \geqslant 12$, once we force the BNP condition on $C$, we are left, in each genus, with only five possible cases for the plane curves $C_{0}$ (one of which, for example, is a plane degree $3 g$ curve with eight points of multiplicity $g$ and one point of multiplicity $g-1$ ), and that in all of these cases we get $Z^{2} \geqslant-6$.

The Brill-Noether-Petri tool to obtain such a drastic reduction of cases is rather rudimentary. We use time and again the pencil of cubic curves passing through eight of the points of maximal multiplicity of $C_{0}$, and we exclude cases by insisting that this pencil should cut out on $C$ a BNP linear series.

Finally, let us say two words about the cases in which $S_{0} \cong \mathbb{P}(E)$ with $E$ a rank 2 bundle over a smooth curve $\Gamma$. The case when the genus of $\Gamma$ is bigger than 1 is solved swiftly via an elementary Brill-Noether argument. The genus zero case is reduced to the case of $\mathbb{P}^{2}$. The genus 1 case proceeds exactly as the case of $\mathbb{P}^{2}$; only, it is much easier. 


\section{ON HYPERPLANE SECTIONS OF K3 SURFACES}

\section{Part I.}

\section{The scroll}

We assume all schemes to be defined over $\mathbb{C}$. We let $C$ be a projective non-singular irreducible curve of genus $g \geqslant 11$. Recall the following definition.

Definition 2.1. Let $L$ be an invertible sheaf on $C$. The natural map

$$
\mu_{0}(L): H^{0}(C, L) \otimes H^{0}\left(C, \omega_{C} L^{-1}\right) \longrightarrow H^{0}\left(C, \omega_{C}\right)
$$

is called the Petri map of $L$. If $\mu_{0}(L)$ is injective, then $L$ is said to be Brill-Noether-Petri. If all $L \in \operatorname{Pic}(C)$ are Petri, then $C$ is Brill-Noether-Petri (for short, BNP).

Remark 2.2. The Brill-Noether number $\rho=g-(r+1)(g-d+r)$ is non-negative for every BNP invertible sheaf $L$ on $C$ such that $\operatorname{deg} L=d$ and $h^{0}(C, L)=r+1$. We will almost always consider the case of base-point-free pencils on $C$. If $V \subset H^{0}(C, L)$ is one such, then

$$
\operatorname{ker}\left\{\mu_{0, V}: V \otimes H^{0}\left(K L^{-1}\right) \longrightarrow H^{0}(C, K)\right\} \cong H^{0}\left(C, K L^{-2}\right) .
$$

Moreover, the condition $\rho \geqslant 0$, for pencils, translates into $2 d \geqslant g+2$.

The following theorem by Voisin [Voi88] is at the centre of our paper.

Theorem 2.3. Assume $\operatorname{Cliff}(C) \geqslant 3$, and let $L$ be a general element of a component $S$ of $W_{g-2}^{1}(C)$. Then

(i) $|L|$ is a primitive $g_{g-2}^{1}$, that is, both $L$ and $\omega_{C} L^{-1}$ are base-point-free;

(ii) $\left|\omega_{C} L^{-1}\right|$ maps $C$ birationally onto a plane curve $\Gamma$ of degree $g$ having only double points as singularities;

(iii) $L$ is $B N P$.

Proof. For parts (i) and (ii), we refer to [Voi88, Proposition II.0].

(iii) If $L$ is not BNP, then $\omega_{C} L^{-2}$ is effective of degree 2 . Since $\operatorname{dim}(S) \geqslant g-6$, the rational map

$$
S-->C^{(2)}, \quad L \longmapsto \omega_{C} L^{-2}
$$

has positive-dimensional fibres, and this implies that $L^{2}$ has infinitely many distinct square roots, which is impossible.

From now on, in Part I, we will assume $\operatorname{Cliff}(C) \geqslant 3$ and we will denote by $L$ an element of $W_{g-2}^{1}(C)$ satisfying conditions (i), (ii) and (iii) of Theorem 2.3. Note that since $g \geqslant 11$, the condition $\operatorname{Cliff}(C) \geqslant 3$ is equivalent to the condition that $C$ does not have a $g_{4}^{1}$ (is not 4-gonal).

In order to simplify the notation, we will identify $C$ with its canonical model

$$
\varphi_{\omega_{C}}(C) \subset \mathbb{P} H^{0}\left(C, \omega_{C}\right)^{\vee} \cong \mathbb{P}^{g-1} .
$$

We will write $\mathbb{P}$ instead of $\mathbb{P}^{g-1}$ whenever no confusion is possible. With the sheaf $L$, there is associated, in a well-known way, a cubic scroll $X \subset \mathbb{P}$ containing $C$ (see, for example, [Sch86]). Let us recall how this is done. We set

$$
V:=H^{0}\left(C, \omega_{C} L^{-1}\right) \cong \mathbb{C}^{3} .
$$

The Petri map

$$
\mu_{0}(L): V \otimes H^{0}(C, L) \longrightarrow H^{0}\left(C, \omega_{C}\right)=H^{0}(\mathbb{P}, \mathcal{O}(1))
$$




\section{E. Arbarello, A. Bruno and E. Sernesi}

produces a $3 \times 2$ matrix $M$ of linear forms on $\mathbb{P}$ whose $2 \times 2$ minors vanish on $C$. Explicitly, let $x_{0}, x_{1}, x_{2}$ be a basis of $H^{0}\left(\omega_{C} L^{-1}\right)$. Let $y_{1}, y_{2}$ be basis of $H^{0}(L)$. Since the Petri map is injective, the six elements $\omega_{i j}=x_{i} y_{j}$ are linearly independent in $H^{0}\left(\omega_{C}\right)$ and can be viewed as linear forms $X_{i j}$ in $\mathbb{P}$. Then

$$
M=\left\{X_{i j}\right\}, \quad X_{i j \mid C}=\omega_{i j}=x_{i} y_{j} .
$$

The variety $X \subset \mathbb{P}$ defined by the minors of $M$ is a cubic scroll of codimension 2 containing $C$.

Choose a $(g-6)$-dimensional subspace $W \subset H^{0}\left(C, K_{C}\right)$ such that

$$
H^{0}\left(C, \omega_{C}\right)=\operatorname{Im}\left(\mu_{0}(L)\right) \oplus W \cong\left(H^{0}(C, L) \otimes V\right) \oplus W .
$$

Consider on $\mathbb{P}^{1}$ the vector bundle

$$
\mathcal{E}:=\left(V \otimes \mathcal{O}_{\mathbb{P}^{1}}(1)\right) \oplus\left(W \otimes \mathcal{O}_{\mathbb{P}^{1}}\right),
$$

and let $\widetilde{X}:=\mathbb{P} \mathcal{E}$. Then $X$ is the image of the morphism

$$
\nu:=\varphi_{\mathcal{O}_{\tilde{X}}(1)}: \widetilde{X} \longrightarrow \mathbb{P}^{g-1} .
$$

It is a cone over the Segre variety $\mathbb{P}^{1} \times \mathbb{P}^{2} \subset \mathbb{P}^{5}$ with a $(g-7)$-dimensional vertex equal to $\mathbb{P} W$. In particular, $X$ is arithmetically Cohen-Macaulay. One has

$$
\nu^{-1}(\mathbb{P} W) \cong \mathbb{P}^{1} \times \mathbb{P} W
$$

while

$$
\nu_{\mid \widetilde{X} \backslash \nu^{-1}(\mathbb{P} W)}: \tilde{X} \backslash \nu^{-1}(\mathbb{P} W) \longrightarrow X \backslash \mathbb{P} W
$$

is an isomorphism. Let $\pi: \widetilde{X} \longrightarrow \mathbb{P}^{1}$ be the natural projection, and let

$$
\widetilde{X} \supset \widetilde{R}=\pi^{-1}(p) \cong \mathbb{P}^{g-4}
$$

be any fibre of $\pi$. Then $\widetilde{R}$ is a Cartier divisor in $\widetilde{X}$ which is mapped isomorphically onto its image $R:=\nu(\widetilde{R}) \subset X$. Note the following:

(a) The image $R \subset X$ is a Weil divisor containing $\mathbb{P} W$ and is not Cartier along $\mathbb{P} W=\operatorname{Sing}(X)$.

(b) Since $R \cap C \in|L|$ and $|L|$ has no fixed points, we have $C \cap \mathbb{P} W=\emptyset$.

(c) Identifying $C$ with $\widetilde{C}:=\nu^{-1}(C) \subset \widetilde{X}$, we have

$$
\mathcal{O}_{\widetilde{C}}(\widetilde{R})=L=\mathcal{O}_{C}(R) .
$$

We shall denote by $H$ a hyperplane section of $X$, and we set

$$
\widetilde{H}:=\nu^{*} H \text {. }
$$

Of course, we have

$$
\mathcal{O}_{\widetilde{X}}(\widetilde{H})=\mathcal{O}_{\widetilde{X}}(1), \quad \mathcal{O}_{C}(H)=\omega_{C}
$$

We will also need the following.

LEMMA 2.4. In the above situation, the following hold:

(i) All quadrics $Q \in H^{0}\left(\mathbb{P}^{g-1}, \mathcal{I}_{X / \mathbb{P}}(2)\right)$ have rank at most 4.

(ii) For each point $p \in X \backslash \mathbb{P} W$, there is a unique (up to a constant factor) non-zero quadric $Q_{p} \in H^{0}\left(\mathbb{P}^{g-1}, \mathcal{I}_{X / \mathbb{P}}(2)\right)$ that is singular at $p$.

(iii) If a quadric $Q \in H^{0}\left(\mathbb{P}, \mathcal{O}_{\mathbb{P}}(2)\right)$ contains $R \cup C$ for some ruling $R \subset X$ and is singular at a point $p \in C$ with $p \notin R$, then $Q=Q_{p} \in H^{0}\left(\mathbb{P}^{g-1}, \mathcal{I}_{X / \mathbb{P}}(2)\right)$. 


\section{ON HYPERPLANE SECTIONS OF K3 SURFACES}

Proof. (i) If $Q, Q^{\prime} \in H^{0}\left(\mathbb{P}, \mathcal{I}_{X / \mathbb{P}}(2)\right)$ are distinct, then $Q \cap Q^{\prime}=X \cup Z$, where $Z \cong \mathbb{P}^{g-3}$. Then $Q$ has rank at most 4 because it contains a $\mathbb{P}^{g-3}$.

(ii) Projecting $X$ from $p$, we get a quadric $\Sigma \subset \mathbb{P}^{g-2}$. Then $Q_{p}$ is the cone projecting $\Sigma$ from $p$.

(iii) The quadric $Q$ contains $R$ and is singular at $p$, thus it contains $\langle p, R\rangle \cong \mathbb{P}^{g-3}$. Hence $\operatorname{rk}(Q) \leqslant 4$. By construction, $Q$ is the quadric associated with the pencil $|L(p)|=|L|+p$, which is a $g_{g-1}^{1}$ (by definition, this is $X\left(g_{g-1}^{1}\right)$, the union of the codimension 2 spaces $\langle D\rangle$, as $D$ runs in $\left.g_{g-1}^{1}\right)$. Then $Q$ contains all the spaces $\left\langle p, R^{\prime}\right\rangle$ as well, and therefore it contains $X$.

\section{A digression on blow-ups}

In this section, we fix some notation and recall some well-known facts.

Let $S$ be a projective non-singular algebraic surface, $P_{0} \in S$ and $c \geqslant 1$ an integer. Let

$$
\sigma: \widetilde{S}=S_{c} \stackrel{\sigma_{c}}{\longrightarrow} \cdots \stackrel{\sigma_{2}}{\longrightarrow} S_{1} \stackrel{\sigma_{1}}{\longrightarrow} S
$$

be the composition of $c$ blow-ups of $S$ with centres $P_{0}$ and $c-1$ points $P_{1}, \ldots, P_{c-1}$ belonging to successive infinitesimal neighbourhoods of $P_{0}$. In other words, $P_{j}$ belongs to the $(-1)$-curve on $S_{j}$ coming from blowing up $P_{j-1} \in S_{j-1}$ for $j=1, \ldots, c-1$. If $j \geqslant 2$, we assume that $P_{j}$ does not also belong to the $(-2)$-curve coming from blowing up $P_{j-2}$.

Notation 3.1. We let $e_{1}, \ldots, e_{c} \subset \widetilde{S}$ be the components of the tree of exceptional rational curves arising from the $c$ blow-ups, where $e_{j}$ is the rational curve coming from the $j$ th blow-up. Then

$$
e_{c}^{2}=-1 ; \quad e_{j}^{2}=-2, \quad j=1, \ldots, c-1 ; \quad e_{j} \cdot e_{j+1}=1 ; \quad j=1, \ldots, c-1,
$$

and $e_{j} \cdot e_{h}=0$ otherwise. Moreover, we let

$$
E_{j}:=\sum_{h=j}^{c} e_{h} .
$$

We have

$$
E_{j}^{2}=-1, \quad E_{i} \cdot E_{j}=0, \quad i \neq j .
$$

With this notation, we have

$$
K_{\widetilde{S}}=\sigma^{*} K_{S}+\sum_{j} E_{j}
$$

Note also that

$$
E_{j} \cdot e_{h}=\left\{\begin{aligned}
1 & \text { if } j=h+1 \\
-1 & \text { if } j=h \\
0 & \text { otherwise }
\end{aligned}\right.
$$

Let $D \subset S$ be an integral curve. Recall that $P \in D$ is called an $A_{n}$-singularity for some $n \geqslant 1$ if a local equation of $D$ at $P$ is analytically equivalent to $y^{2}+x^{n+1}=0$. All $A_{n}$-singularities are double points and conversely every double point is an $A_{n}$-singularity for a unique $n$. The delta-invariant of an $A_{n}$-singularity is [GLS07, I.3.4, p. 206, following Remark 3.31.1]

$$
\delta=\left[\frac{n+1}{2}\right] \text {. }
$$




\section{E. Arbarello, A. Bruno and E. Sernesi}

Let $\mathbf{c} \subset \mathcal{O}_{D, P}$ be the conductor ideal, that is, the annihilator of $\overline{\mathcal{O}}_{D, P} / \mathcal{O}_{D, P}$, where $\overline{\mathcal{O}}_{D, P}$ denotes the integral closure of $\mathcal{O}_{D, P}$. Then $\operatorname{dim}_{\mathbb{C}} \mathcal{O}_{D, P} / \mathbf{c}=\delta$. Let $\mathbf{A} \subset \mathcal{O}_{S, P}$ be the inverse image of $\mathbf{c}$ under $\mathcal{O}_{S, P} \rightarrow \mathcal{O}_{D, P}(\mathbf{A}$ is the local adjoint ideal of $D$ at $P$ ). Since it has the same colength $\delta$ in $\mathcal{O}_{S, P}$ as $\mathbf{c} \subset \mathcal{O}_{D, P}$ and contains the jacobian ideal of $D$ at $P$ [DH88], the local adjoint ideal of an $A_{n}$-singularity is analytically equivalent to

$$
\left(x^{\delta}, y\right)=\left(x^{[(n+1) / 2]}, y\right) .
$$

In particular, it defines a curvilinear scheme of colength $\delta$. An $A_{n}$-singularity of $D$ at $P$ can be resolved by precisely $\delta$ blow-ups [GLS07, Proposition 3.34]. After performing the appropriate blow-ups of $S$, we end up with a diagram

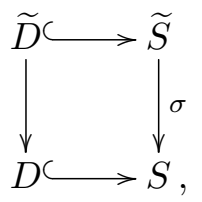

where $\widetilde{D}$ is the proper transform of $D$ and $\widetilde{S}$ contains a tree of rational curves exactly analogous to the one described above, with $c$ replaced by $\delta$. Moreover,

$$
\begin{gathered}
\widetilde{D}=\sigma^{*} D-2 \sum_{j} E_{j} \\
\widetilde{D} \cdot e_{\delta}>0, \quad \widetilde{D} \cdot e_{j}=0, \quad j=1, \ldots, \delta-1 .
\end{gathered}
$$

The last assertions follow from the fact that the total transform of an $A_{n}$-singularity for $n \geqslant 3$ is a $D_{n+1}$-singularity (see [BPvdV84, II, proof of Theorem (8.1)]). Finally, observe that if $\delta \geqslant 2$, the union

$$
\bigcup_{j=1}^{\delta-1} e_{j}
$$

is a Hirzebruch-Jung configuration and therefore it can be contracted to a rational double point [BPvdV84, III, Proposition 3.1].

\section{The surface $P$}

The plane curve $\Gamma:=\varphi_{\omega_{C} L^{-1}}(C) \subset \mathbb{P}^{2}$ is irreducible of degree $g$ and has only double points $P_{1}, \ldots, P_{k}$ as singularities, by Theorem 2.3. For $i=1, \ldots, k$, the point $P_{i}$ is an $A_{n_{i}}$-singularity for $\Gamma$, for some $n_{i}$. Let $\delta_{i}=\left[\left(n_{i}+1\right) / 2\right]$ be the corresponding local delta-invariant. Then

$$
\sum_{i} \delta_{i}=: \delta=\left(\begin{array}{c}
g-1 \\
2
\end{array}\right)-g .
$$

By what we have seen in Section 3, the adjoint ideal sheaf $\operatorname{Adj}(\Gamma) \subset \mathcal{O}_{\mathbb{P}^{2}}$ defines a curvilinear scheme. Let $Y \subset \mathbb{P}^{2}$ be a general adjoint curve to $\Gamma$ of degree $g-4$. Since $|L|$ is a base-point-free pencil, $Y$ is non-singular at $P_{1}, \ldots, P_{k}$. We have a diagram

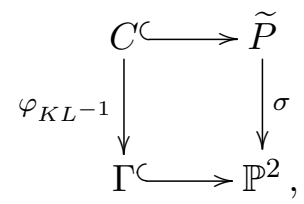




\section{ON HYPERPLANE SECTIONS OF K3 SURFACES}

where $\sigma$ is a sequence of $\delta$ blow-ups and $C$ is the proper transform of $\Gamma$. We will denote by $e_{i 1}, \ldots, e_{i \delta_{i}}$ the components of $\sigma^{-1}\left(P_{i}\right)$ and set

$$
E_{i j}=\sum_{h=j}^{\delta_{i}} e_{i h}
$$

For the general theory of adjoint curves, we refer to Szpiro's book [Szp79].

Proposition 4.1. The line bundle $\mathcal{O}_{\widetilde{P}}\left(K_{\widetilde{P}}+C\right)$ is globally generated and maps $\widetilde{P}$ birationally onto a surface $P \subset \mathbb{P}$ obtained by contracting all the (-2)-curves of $\widetilde{P}$. In particular, $P$ is normal and has at most rational double points. If $\Gamma$ is nodal, then $P \cong \widetilde{P}$ is non-singular. Furthermore, $P \subset X$.

Proof. Let $E=\sum E_{i j}$, and let $\widetilde{Y} \subset \widetilde{P}$ be the proper transform of $Y$. We have

$$
\begin{aligned}
K_{\widetilde{P}} & =\sigma^{*} \mathcal{O}_{\mathbb{P}^{2}}(-3)(E), \\
C & =\sigma^{*} \Gamma-2 E=\sigma^{*} \mathcal{O}_{\mathbb{P}^{2}}(g)(-2 E), \\
K_{\widetilde{P}}+C & \sim \sigma^{*} \mathcal{O}_{\mathbb{P}^{2}}(g-3)(-E), \\
\widetilde{Y} & \sim \sigma^{*} \mathcal{O}_{\mathbb{P}^{2}}(g-4)(-E) .
\end{aligned}
$$

Let $\Lambda \subset \mathbb{P}^{2}$ be a line, and let $\lambda \subset \widetilde{P}$ be its proper transform in $\widetilde{P}$. Assume first that $\Lambda$ does not contain any of the points $P_{1}, \ldots, P_{k}$. Since $\left|\omega_{C} L^{-1}\right|$ is a complete $g_{g}^{2}$, the adjoint ideal to $\Gamma$ imposes independent conditions to the curves of degree at least $g-4$. This is an exercise in [ACGH85, Appendix A] in the nodal case. In the general case, this follows from the general theory of adjoints as explained in [Szp79, II.9, pp. 40-42]. As a result, the exact sequence

$$
0 \longrightarrow \sigma^{*} \mathcal{O}_{\mathbb{P}^{2}}(g-4)(-E) \longrightarrow \sigma^{*} \mathcal{O}_{\mathbb{P}^{2}}(g-3)(-E) \longrightarrow \mathcal{O}_{\lambda}(g-3) \longrightarrow 0
$$

is exact on global sections, and therefore $\left|K_{\widetilde{P}}+C\right|$ embeds $\lambda$. Assume now that $\Lambda$ contains $P_{1}$ and none of the $P_{i}$ for $i \geqslant 2$. We have

$$
\mathcal{O}_{\widetilde{P}}(\lambda)=\sigma^{*} \mathcal{O}_{\mathbb{P}^{2}}(1)\left(-\sum_{j \leqslant j_{0}} E_{1 j}\right)
$$

for some $j_{0} \geqslant 1$ and

$$
E \cdot \sum_{j \leqslant j_{0}} E_{1 j}=-j_{0}
$$

Consider the exact sequence

$$
0 \longrightarrow \sigma^{*} \mathcal{O}_{\mathbb{P}^{2}}(g-4)\left(-E+\sum_{j \leqslant j_{0}} E_{1 j}\right) \longrightarrow \sigma^{*} \mathcal{O}_{\mathbb{P}^{2}}(g-3)(-E) \longrightarrow \mathcal{O}_{\lambda}\left(g-3-j_{0}\right) \longrightarrow 0 .
$$

As $C \cdot \lambda \geqslant 0$ ( $C$ is irreducible) and $C \cdot \lambda=g-2 j_{0}$, we get $g \geqslant 2 j_{0}$. On the other hand, $g \geqslant 11$, so that $g-3-j_{0}>0$. Since $h^{0}\left(\widetilde{P}, \sigma^{*} \mathcal{O}_{\mathbb{P}^{2}}(g-4)\left(-E+\sum_{j \leqslant j_{0}} E_{1 j}\right)\right)=2+j_{0}$, the above sequence is exact on global sections. A similar argument can be given for any other line $\Lambda \subset \mathbb{P}^{2}$, no matter how many of the $P_{i}$ it contains. This implies that $\left|K_{\widetilde{P}}+C\right|$ is very ample on the proper transform $\lambda$ of any line $\Lambda \subset \mathbb{P}^{2}$. Therefore $\left|K_{\widetilde{P}}+C\right|$ is base-point-free and very ample on $\widetilde{P} \backslash \bigcup e_{i j}$. Let us prove it is also base-point-free on $\bigcup e_{i j}$.

Case 1: The linear system $\left|K_{\widetilde{P}}+C\right|$ has no base point on $e_{i, \delta_{i}}$. Set $C \cdot e_{i, \delta_{i}}=q_{1}+q_{2}$. If there were a base point for $\left|K_{\widetilde{P}}+C\right|$, this could not lie on $C$ as $\left|K_{\widetilde{P}}+C\right|$ cuts out the canonical series on $C$. But then, since $\left(K_{\widetilde{P}}+C\right) \cdot e_{i, \delta_{i}}=1$, any element of $\left|K_{\widetilde{P}}+C\right|$ passing through $q_{1}$ 


\section{E. Arbarello, A. Bruno and E. Sernesi}

would pass through $q_{2}$ (indeed would contain $e_{i, \delta_{i}}$ as a component) which is absurd, since $C$ is non-hyperelliptic.

Case 2: The linear system $\left|K_{\widetilde{P}}+C\right|$ has no base point on $e_{i, j}$ with $j<\delta_{i}$. We know that $\left(K_{\widetilde{P}}+C\right) \cdot e_{i, j}=0$ if $j<\delta_{i}$, so that $\left|K_{\widetilde{P}}+C\right|$ has a base point on $e_{i, j}$ only if $e_{i, j}$ is a fixed component of $\left|K_{\widetilde{P}}+C\right|$. But then $\left|K_{\widetilde{P}}+C\right|$ has a fixed point on $e_{i, j+1}$ and so on, until one reaches $e_{i, \delta_{i}}$ and a contradiction.

It remains to check how $\left|K_{\widetilde{P}}+C\right|$ restricts to the curves $E_{i j}$. Using (3.1), we deduce that

$$
\left(K_{\widetilde{P}}+C\right) \cdot e_{i j}= \begin{cases}1 & \text { if } j=\delta_{i}, \\ 0 & \text { if } 1 \leqslant j \leqslant \delta_{i}-1 .\end{cases}
$$

Therefore $\left|K_{\widetilde{P}}+C\right|$ contracts the Hirzebruch-Jung chains $e_{i 1}+\cdots+e_{i \delta_{i}-1}$ of $(-2)$-curves to rational double points and maps the $(-1)$-curves $e_{i \delta_{i}}$ isomorphically onto lines. Then the morphism

$$
\Phi: \widetilde{P} \longrightarrow \mathbb{P}^{g-1}
$$

defined by the linear system $\left|K_{\widetilde{P}}+C\right|$ maps $\widetilde{P}$ onto a surface $P$ containing $C$ and having the asserted properties. Finally, let us show that $P$ is contained in $X$. Let $x_{0}, x_{1}, x_{2}$ be a basis of $H^{0}\left(\omega_{C} L^{-1}\right)$, and recall the matrix $M$ introduced in (2.2). Let $\phi_{1}=\phi_{1}\left(x_{0}, x_{1}, x_{2}\right)$ and $\phi_{2}=\phi_{2}\left(x_{0}, x_{1}, x_{2}\right)$ be degree $g-4$ adjoint polynomials such that $\phi_{1 \mid C}=y_{1}, \phi_{2 \mid C}=y_{2}$ is a basis of $H^{0}(L)$. Under the morphism $\Phi: \widetilde{P} \rightarrow \mathbb{P}$, we get $\Phi^{*}\left(X_{i j}\right)=x_{i} \phi_{j}$. This means that the matrix $M$ has rank 1 on $P$ or, equivalently, that $P$ is contained in $X$. This concludes the proof.

For each ruling $R \subset X$, we will set

$$
Y_{R}:=R \cap P \quad \text { and } \quad \widetilde{Y}_{R}:=\Phi^{-1}\left(Y_{R}\right) \subset \widetilde{P} .
$$

Note that the curves $\widetilde{Y}_{R}$ are the proper transforms of the adjoint curves to $\Gamma$ of degree $g-4$. In particular,

$$
\mathcal{O}_{C}\left(\widetilde{Y}_{R}\right)=L
$$

Lemma 4.2. $h^{0}\left(X, \mathcal{I}_{C \cup R / X}(2 H)\right) \geqslant h^{0}\left(X, \mathcal{I}_{P \cup R / X}(2 H)\right) \geqslant g-5$.

Proof. The first inequality is obvious. From the exact sequence

$$
0 \longrightarrow \mathcal{I}_{X / \mathbb{P}}(2) \longrightarrow \mathcal{I}_{R / \mathbb{P}}(2) \longrightarrow \mathcal{I}_{R / X}(2 H) \longrightarrow 0,
$$

we deduce

$$
\begin{aligned}
h^{0}\left(X, \mathcal{I}_{R / X}(2 H)\right) & \geqslant h^{0}\left(\mathbb{P}, \mathcal{I}_{R / \mathbb{P}}(2)\right)-h^{0}\left(\mathbb{P}, \mathcal{I}_{X / \mathbb{P}}(2)\right) \\
& =(3 g-3)-3=3 g-6 .
\end{aligned}
$$

Now, consider the exact sequence

$$
0 \longrightarrow \mathcal{I}_{P \cup R / X}(2 H) \longrightarrow \mathcal{I}_{R / X}(2 H) \longrightarrow \mathcal{I}_{Y_{R} / P}(2 H) \longrightarrow 0,
$$

which implies

$$
h^{0}\left(X, \mathcal{I}_{P \cup R / X}(2 H)\right) \geqslant 3 g-6-h^{0}\left(P, \mathcal{I}_{Y_{R} / P}(2 H)\right) .
$$


Finally, observe that

$$
\begin{aligned}
h^{0}\left(P, \mathcal{I}_{Y_{R} / P}(2 H)\right) & \leqslant h^{0}\left(\widetilde{P}, \mathcal{O}_{\widetilde{P}}\left(2 H-\widetilde{Y}_{R}\right)\right) \\
& \leqslant h^{0}\left(C, \omega_{C}^{2} L^{-1}\right) \\
& =2 g-1 .
\end{aligned}
$$

The first inequality is obvious, and the second inequality follows from the exact sequence

$$
0 \longrightarrow \mathcal{O}_{\widetilde{P}}\left(2 H-\widetilde{Y}_{R}-C\right) \longrightarrow \mathcal{O}_{\widetilde{P}}\left(2 H-\widetilde{Y}_{R}\right) \longrightarrow \omega_{C}^{2} L^{-1} \longrightarrow 0
$$

and from the fact that $H^{0}\left(\widetilde{P}, \mathcal{O}_{\widetilde{P}}\left(2 H-\widetilde{Y}_{R}-C\right)\right)=0$.

Proposition 4.3. The surface $P$ is non-singular along $C$.

Proof. Keeping the notation of Proposition 4.1, the curve $C \subset \widetilde{P}$ meets the $(-1)$-curves $e_{i \delta_{i}}$ and does not meet the other $e_{i j}$ because $C \cup e_{i \delta_{i}} \cup e_{i \delta_{i}-1}$ is the total transform of a $D_{4}$ or $D_{5}$ singularity [BPvdV84, II, proof of Theorem (8.1)]. This implies that $C$ does not contain any of the singular points of $P$.

\section{Triviality of a (restricted) conormal sheaf}

If $A \subset B$, we shall adopt the standard notation $N_{A / B}^{\vee}$ for the conormal sheaf $\mathcal{I}_{A / B} / \mathcal{I}_{A / B}^{2}$ of $A$ in $B$.

Lemma 5.1. The sheaf $N_{P / X \mid C}^{\vee}=N_{P / X}^{\vee} \otimes \mathcal{O}_{C}$ is locally free and

$$
c_{1}\left(N_{P / X \mid C}^{\vee}(2 H-L)\right)=0 .
$$

Proof. By Proposition 4.3, the conormal sheaf $N_{P / X}^{\vee}$ is locally free of rank $g-5$ along $C$. Consider the exact sequence of locally free sheaves on $C$

$$
0 \longrightarrow N_{C / X}^{\vee}(2 H-L) \longrightarrow \Omega_{X \mid C}^{1}(2 H-L) \longrightarrow \omega_{C}(2 H-L) \longrightarrow 0 .
$$

We have

$$
\begin{aligned}
c_{1}\left(N_{C / X}^{\vee}(2 H-L)\right) & =c_{1}\left(\Omega_{X \mid C}^{1}(2 H-L)\right)-c_{1}\left(\omega_{C}(2 H-L)\right) \\
& =c_{1}\left(\Omega_{\widetilde{X} \mid C}^{1}(2 H-L)\right)-c_{1}\left(\omega_{C}(2 H-L)\right) \\
& =-(g-3) H+L+(g-3)(2 H-L)-(3 H-L) \\
& =(g-6) H-(g-5) L \\
& =(g-6) \omega_{C}-(g-5) L .
\end{aligned}
$$

On the other hand, since $P$ is non-singular along $C$, we have the exact sequence on $C$

$$
0 \longrightarrow N_{P / X \mid C}^{\vee}(2 H-L) \longrightarrow N_{C / X}^{\vee}(2 H-L) \longrightarrow N_{C / P}^{\vee}(2 H-L) \longrightarrow 0,
$$

and therefore

$$
\begin{aligned}
c_{1}\left(N_{P / X \mid C}^{\vee}(2 H-L)\right) & =c_{1}\left(N_{C / X}^{\vee}(2 H-L)\right)-c_{1}\left(N_{C / P}^{\vee}(2 H-L)\right) \\
& =(g-6) \omega_{C}-(g-5) L-\left(2 \omega_{C}-L-\mathcal{O}_{C}(C)\right) \\
& =(g-8) \omega_{C}-(g-6) L+\mathcal{O}_{C}(C) .
\end{aligned}
$$




\section{E. Arbarello, A. Bruno and E. Sernesi}

We can rewrite this class as the restriction of a class on $\widetilde{P}$ by letting

$$
\ell:=\sigma^{*} \mathcal{O}_{\mathbb{P}^{2}}(1) .
$$

With this notation, we have

$$
\omega_{C}=\mathcal{O}_{C}((g-3) \ell-E), \quad L=\mathcal{O}_{C}((g-4) \ell-E), \quad \mathcal{O}_{C}(C)=\mathcal{O}_{C}(g \ell-2 E),
$$

where $E$ has the same meaning as in the proof of Proposition 4.1. Therefore the class

$$
c_{1}\left(N_{P / X \mid C}^{\vee}(2 H-L)\right)
$$

is the restriction to $C$ of the class on $\widetilde{P}$ :

$$
(g-8)((g-3) \ell-E)-(g-6)((g-4) \ell-E)+(g \ell-2 E)=0 .
$$

Now, we can prove the following triviality result.

Proposition 5.2. The restriction homomorphism

$$
\mathcal{I}_{P \cup R / X}(2 H) \longrightarrow N_{P / X \mid C}^{\vee}(2 H-L)
$$

induces an isomorphism

$$
\bar{\alpha}: H^{0}\left(X, \mathcal{I}_{P \cup R / X}(2 H)\right) \otimes \mathcal{O}_{C} \stackrel{\cong}{\longrightarrow} N_{P / X \mid C}^{\vee}(2 H-L) .
$$

Proof. By Proposition 4.3, the sheaf $N_{P / X \mid C}^{\vee}(2 H-L)$ is locally free of rank $g-5$. Choose $p \in C$ but $p \notin R$. Consider the vector space homomorphism

$$
\bar{\alpha}_{p}: H^{0}\left(X, \mathcal{I}_{P \cup R / X}(2 H)\right) \longrightarrow N_{P / X \mid C}^{\vee}(2 H-L)_{p} \otimes \mathbb{C} .
$$

The homomorphism $\bar{\alpha}_{p}$ fits into the following commutative and exact diagram

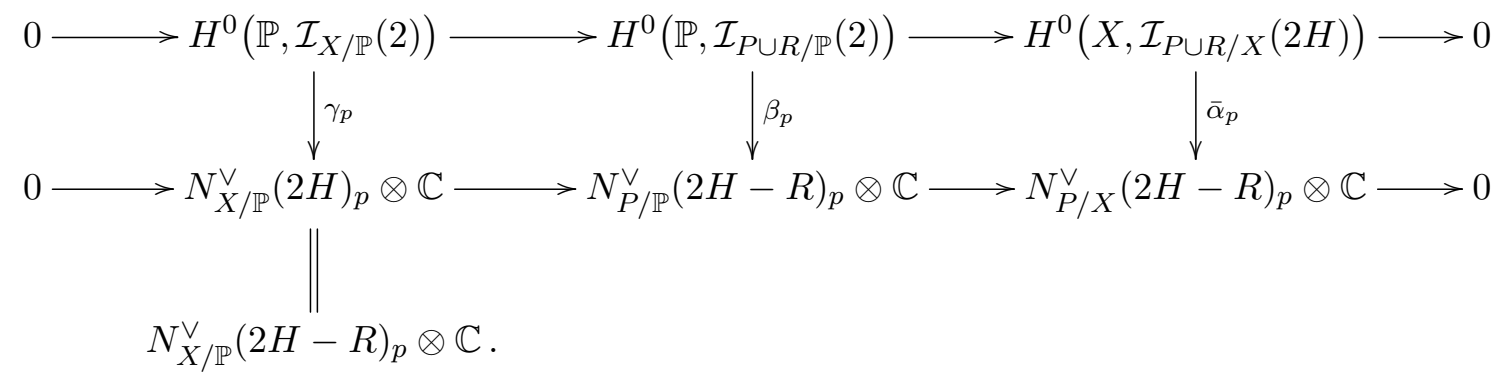

The second row is exact since $N_{P / X}^{\vee}$ is locally free along $C$. The first row is exact because $H^{1}\left(\mathbb{P}, \mathcal{I}_{X / \mathbb{P}}(2)\right)=0$. The homomorphism $\gamma_{p}$ is surjective since $p$ is a smooth point of $X$ and $X$ is cut out by quadrics. It follows that there is a surjection

$$
\rho: \operatorname{ker}\left(\beta_{p}\right) \longrightarrow \operatorname{ker}\left(\bar{\alpha}_{p}\right) \text {. }
$$

Now, let $Q \in \operatorname{ker}\left(\bar{\alpha}_{p}\right)$ and lift $Q$ to $Q^{\prime} \in \operatorname{ker}\left(\beta_{p}\right)$. This means that $Q^{\prime}$ is singular at $p$ and contains $R \cup C$. Then $Q^{\prime}$ contains $X$, by Lemma 2.4(iii), and therefore $Q=\rho\left(Q^{\prime}\right)=0$. Thus $\bar{\alpha}_{p}$ is injective.

Recall (Lemma 4.2) that $h^{0}\left(X, \mathcal{I}_{P \cup R / X}(2 H)\right) \geqslant g-5$. Therefore $\bar{\alpha}_{p}$ is an isomorphism. Thus $\bar{\alpha}$ is a morphism between a trivial bundle of rank $g-5$ and a locally free rank $g-5$ bundle with trivial Chern class. Since for a point $p \in C$, the homomorphism $\bar{\alpha}_{p}$ is an isomorphism, $\bar{\alpha}$ is an isomorphism as well. 


\section{ON HYPERPLANE SECTIONS OF K3 SURFACES}

\section{The square of the ideal sheaf of $C \subset X$}

The purpose of this section is to prove the following proposition.

Proposition 6.1. For all $k \geqslant 3$, we have $H^{1}\left(X, \mathcal{I}_{C / X}^{2}(k H)\right)=0$.

Proof. We present the proof in the case $k=3$, leaving to the reader the straightforward generalisation to the case $k \geqslant 4$. Consider the following diagram:

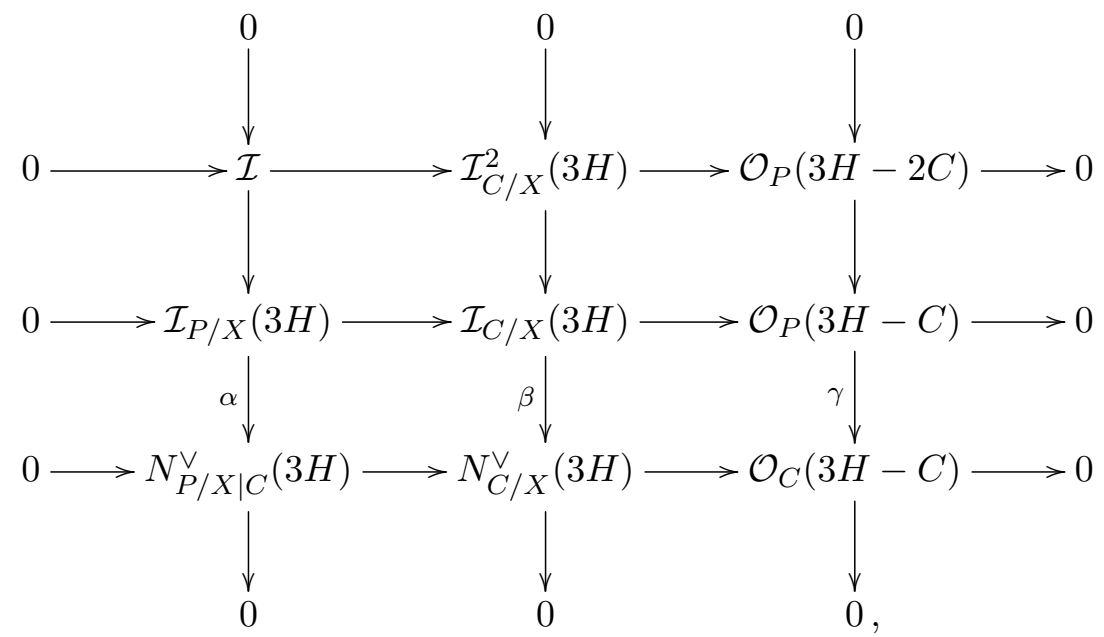

where $\mathcal{I} \subset \mathcal{O}_{X}(3 H)$ is a sheaf defined by the diagram.

Claim. (a) $H^{1}\left(X, \mathcal{I}_{C / X}(3 H)\right)=0$,

(b) $H^{1}\left(P, \mathcal{O}_{P}(3 H-2 C)\right)=0$,

(c) $H^{1}\left(X, \mathcal{I}_{P / X}(3 H)\right)=0$.

Proof. (a) This is a consequence of the fact that $X$ is arithmetically Cohen-Macaulay and $C$ is projectively normal.

(b) Let $\widetilde{H}=\Phi^{*} H$. Then we have

$$
\begin{aligned}
H^{1}\left(\widetilde{P}, \mathcal{O}_{\widetilde{P}}(3 \widetilde{H}-2 C)\right) & =H^{1}\left(\widetilde{P}, 3 K_{\widetilde{P}}+C\right) \\
& =H^{1}\left(\widetilde{P}, \sigma^{*} \mathcal{O}_{\mathbb{P}^{2}}(g-9)+E\right) \\
& =H^{1}\left(\mathbb{P}^{2}, \mathcal{O}_{\mathbb{P}^{2}}(g-9)\right)=0,
\end{aligned}
$$

because $\sigma_{*} \mathcal{O}(E)=\mathcal{O}_{\mathbb{P}^{2}}$ and $R^{1} \sigma_{*} \mathcal{O}(E)=0$. Moreover, we have

$$
H^{1}\left(P, \mathcal{O}_{P}(3 H-2 C)\right)=H^{1}\left(\widetilde{P}, \mathcal{O}_{\widetilde{P}}(3 \widetilde{H}-2 C)\right) .
$$

In fact, by Proposition 4.1 , we get $\Phi_{*} \mathcal{O}_{\widetilde{P}}=\mathcal{O}_{P}$ and $R^{1} \Phi_{*} \mathcal{O}_{\widetilde{P}}=0$.

(c) Consider the exact sequences

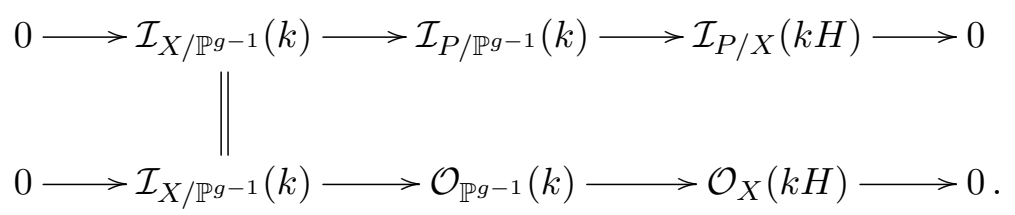

From the bottom one and the CM property of $X$, we deduce

$$
H^{1}\left(\mathbb{P}^{g-1}, \mathcal{I}_{X / \mathbb{P}^{g-1}}(k)\right)=H^{2}\left(\mathbb{P}^{g-1}, \mathcal{I}_{X / \mathbb{P}^{g-1}}(k)\right)=0 \quad \text { for all } k .
$$




\section{E. Arbarello, A. Bruno and E. Sernesi}

From the horizontal one and from $\left(c_{2}\right)$, we deduce

$$
H^{1}\left(X, \mathcal{I}_{P / X}(k H)\right) \cong H^{1}\left(\mathbb{P}^{g-1}, \mathcal{I}_{P / \mathbb{P}^{g-1}}(k)\right) \text { for all } k .
$$

Therefore, for proving claim (c), it suffices to prove that $\mathcal{I}_{P / \mathbb{P}^{g-1}}$ is 3-regular, that is, that

$$
H^{i}\left(\mathbb{P}^{g-1}, \mathcal{I}_{P / \mathbb{P} g-1}(3-i)\right)=0 \quad \text { for all } i>0
$$

(see, for example, [Ser06, Proposition 4.1.1]).

First, suppose $i=1$. The second row of (6.1) tells us that

$$
\begin{aligned}
h^{0}\left(X, \mathcal{I}_{P / X}(2 H)\right) & \geqslant h^{0}\left(X, \mathcal{I}_{C / X}(2 H)\right)-h^{0}\left(\mathcal{O}_{P}(2 H-C)\right) \\
& =\left(\begin{array}{c}
g-2 \\
2
\end{array}\right)-3-\left(\begin{array}{c}
g-4 \\
2
\end{array}\right)=2 g-10,
\end{aligned}
$$

while the first column of (6.1) gives

$$
h^{0}\left(X, \mathcal{I}_{P / X}(2 H)\right) \leqslant h^{0}\left(C, N_{P / X \mid C}^{\vee}(2 H)\right)+h^{0}(X, \mathcal{I}(-1))=2 g-10+0=2 g-10
$$

because $N_{P / X \mid C}^{\vee}(2 H) \cong L^{\oplus g-5}$ and because $h^{0}\left(X, \mathcal{I}_{C / X}^{2}(2 H)\right)=0$, since a quadric cannot be singular along the non-degenerate $C$. Therefore $h^{0}\left(X, \mathcal{I}_{P / X}(2 H)\right)=2 g-10$. Since $H^{1}\left(X, \mathcal{I}_{C / X}(2 H)\right)$ $=0$, we obtain $H^{1}\left(X, \mathcal{I}_{P / X}(2 H)\right)=0$. Finally, by $\left(c_{3}\right)$, we have

$$
H^{1}\left(\mathbb{P}^{g-1}, \mathcal{I}_{P / \mathbb{P}^{g-1}}(2)\right)=H^{1}\left(X, \mathcal{I}_{P / X}(2 H)\right)=0 .
$$

This takes care of the case $i=1$. The remaining cases $i \geqslant 2$ follow easily from the exact sequences

$$
0 \longrightarrow \mathcal{I}_{P / \mathbb{P}^{g-1}}(3-i) \longrightarrow \mathcal{O}_{\mathbb{P}^{g-1}}(3-i) \longrightarrow \mathcal{O}_{P}((3-i) H) \longrightarrow 0
$$

From claim (a), it follows that the proposition is equivalent to the surjectivity of $H^{0}(\beta)$. From claims (b) and (c), we deduce that $H^{0}(\beta)$ is surjective if $H^{0}(\alpha)$ is surjective, where

$$
H^{0}(\alpha): H^{0}\left(X, \mathcal{I}_{P / X}(3 H)\right) \longrightarrow H^{0}\left(N_{P / X \mid C}^{\vee}(3 H)\right) \cong H^{0}\left(X, \mathcal{I}_{P \cup R / X}(2 H)\right) \otimes H^{0}\left(C, \omega_{C} L\right) .
$$

Here, we use Proposition 5.2 to get the isomorphism

$$
N_{P / X \mid C}^{\vee}(3 H) \cong H^{0}\left(X, \mathcal{I}_{P \cup R / X}(2 H)\right) \otimes \omega_{C} L .
$$

Recall that the morphism $\nu: \widetilde{X} \longrightarrow X$ induces an isomorphism

$$
\nu_{\mid \widetilde{U}}: \widetilde{U} \longrightarrow U
$$

where $U=X \backslash \mathbb{P} W$ and $\widetilde{U}=\widetilde{X} \backslash \nu^{-1}(\mathbb{P} W)$.

Claim. (d) We have isomorphisms

$$
H^{0}\left(U, \mathcal{O}_{X}(H+R)\right) \cong H^{0}\left(\widetilde{U}, \mathcal{O}_{\widetilde{X}}(\widetilde{H}+\widetilde{R})\right) \cong H^{0}\left(C, \omega_{C} L\right)
$$

Proof. The first isomorphism is obvious. The second isomorphism is given by the composition

$$
H^{0}\left(\widetilde{U}, \mathcal{O}_{\widetilde{X}}(\widetilde{H}+\widetilde{R})\right) \longrightarrow H^{0}\left(\widetilde{X}, \mathcal{O}_{\widetilde{X}}(\widetilde{H}+\widetilde{R})\right) \longrightarrow H^{0}\left(C, \omega_{C} L\right)
$$

Here the first arrow is the inverse of the restriction and is an isomorphism because $\widetilde{X} \backslash \widetilde{U}$ has high codimension. The second arrow too is an isomorphism. This follows from the exact sequence

$$
0 \longrightarrow \mathcal{I}_{C / \widetilde{X}}(\widetilde{H}+\widetilde{R}) \longrightarrow \mathcal{O}_{\widetilde{X}}(\widetilde{H}+\widetilde{R}) \longrightarrow \omega_{C} L \longrightarrow 0
$$


and the fact that $h^{0}\left(\widetilde{X}, \mathcal{O}_{\widetilde{X}}(\widetilde{H}+\widetilde{R})\right)=2 g-3=h^{0}\left(C, \omega_{C} L\right)$, a computation that follows from the sequence

$$
0 \longrightarrow \mathcal{O}_{\widetilde{X}}(\widetilde{H}) \longrightarrow \mathcal{O}_{\widetilde{X}}(\widetilde{H}+\widetilde{R}) \longrightarrow \mathcal{O}_{\widetilde{R}}(\widetilde{H}) \longrightarrow 0
$$

and the fact that $H^{0}\left(\widetilde{X}, \mathcal{I}_{C / \widetilde{X}}(\widetilde{H}+\widetilde{R})\right)=0$. This last equality follows from the exact sequence

$$
0 \longrightarrow \mathcal{I}_{C / \widetilde{X}}(\widetilde{H}) \longrightarrow \mathcal{I}_{C / \widetilde{X}}(\widetilde{H}+\widetilde{R}) \longrightarrow \mathcal{I}_{C \cap \widetilde{R} / \widetilde{R}}(\widetilde{H}) \longrightarrow 0
$$

From claim (d), we deduce the following commutative diagram:

$$
\begin{aligned}
H^{0}\left(X, \mathcal{I}_{P \cup R / X}(2 H)\right) \otimes H^{0}\left(U, \mathcal{O}_{X}(H+R)\right) & \longrightarrow H^{0}\left(X, \mathcal{I}_{P / X}(3 H)\right) \\
\cong & \downarrow^{0}(\alpha) \\
H^{0}\left(C, N_{P / X \mid C}^{\vee}(2 H-R)\right) \otimes H^{0}\left(C, \omega_{C} L\right) & =\vec{H}^{0}\left(C, N_{P / X \mid C}^{\vee}(3 H)\right),
\end{aligned}
$$

where the left vertical arrow is an isomorphism. This implies the surjectivity of $H^{0}(\alpha)$ and concludes the proof of Proposition 6.1.

\section{The square of the ideal sheaf of $X \subset \mathbb{P}^{g-1}$}

We begin this section with two preliminary lemmas.

Lemma 7.1. Let $r \geqslant 1$, and let $Z:=\mathbb{P}^{1} \times \mathbb{P}^{r} \subset \mathbb{P}^{2 r+1}$ by the Segre embedding. Then

$$
H^{1}\left(Z, \mathcal{I}_{Z / \mathbb{P}^{2 r+1}}^{2}(k)\right)=0
$$

for all $k$.

Proof. For $k \neq 2$, the result is already proved in [Wah97, (1.3.2) and Proposition 4.4]. Moreover, we know that

where

$$
H^{1}\left(Z, \mathcal{I}_{Z / \mathbb{P}^{2 r+1}}^{2}(2)\right)=\operatorname{ker}\left(\Phi_{H}\right)
$$

$$
\Phi_{H}: \wedge^{2} H^{0}\left(Z, \mathcal{O}_{Z}(H)\right) \longrightarrow H^{0}\left(Z, \Omega_{Z}^{1}(2 H)\right)
$$

is the gaussian map of $H=\mathcal{O}_{Z}(1)$ [Wah97, Proposition 1.8]. Therefore it suffices to prove that $\Phi_{H}$ is an isomorphism.

Set $E=H^{0}\left(\mathbb{P}^{1}, \mathcal{O}_{\mathbb{P}^{1}}(1)\right)$ and $F=H^{0}\left(\mathbb{P}^{r}, \mathcal{O}_{\mathbb{P}^{r}}(1)\right)$, and consider the projections

$$
\pi_{1}: Z \longrightarrow \mathbb{P}^{1}, \quad \pi_{2}: Z \longrightarrow \mathbb{P}^{r} .
$$

Then we have

$$
H^{0}\left(Z, \mathcal{O}_{Z}(H)\right)=E \otimes F
$$

and a direct sum decomposition

$$
\wedge^{2}(E \otimes F)=\left(\wedge^{2} E \otimes S^{2} F\right) \oplus\left(S^{2} E \otimes \wedge^{2} F\right) .
$$

On the other hand, we have

$$
\Omega_{Z}^{1}(2 H)=\left(\pi_{1}^{*} \Omega_{\mathbb{P}^{1}}^{1}\right)(2 H) \oplus\left(\pi_{2}^{*} \Omega_{\mathbb{P}^{r}}^{1}\right)(2 H)
$$

and from Künneth formula, we get

$$
H^{0}\left(Z, \Omega_{Z}^{1}(2 H)\right)=\left[H^{0}\left(\mathbb{P}^{1}, \Omega_{\mathbb{P}^{1}}^{1}(2)\right) \otimes S^{2} F\right] \oplus\left[S^{2} E \otimes H^{0}\left(\mathbb{P}^{r}, \Omega_{\mathbb{P}^{r}}^{1}(2)\right)\right] .
$$




\section{E. Arbarello, A. Bruno and E. Sernesi}

Comparing (7.1) and (7.2), we see that the homomorphism

$$
\Phi_{H}: \wedge^{2}(E \otimes F) \longrightarrow H^{0}\left(Z, \Omega_{Z}^{1}(2 H)\right)
$$

decomposes as the sum of the two homomorphisms

$$
\left[\Phi_{\mathcal{O}_{\mathbb{P}^{1}}(1)}\right] \otimes 1_{S^{2} F}: \wedge^{2} E \otimes S^{2} F \longrightarrow H^{0}\left(\mathbb{P}^{1}, \Omega_{\mathbb{P}^{1}}^{1}(2)\right) \otimes S^{2} F
$$

and

$$
1_{S^{2} E} \otimes \Phi_{\mathcal{O}_{\mathbb{P}^{r}(1)}}: S^{2} E \otimes \wedge^{2} F \longrightarrow S^{2} E \otimes H^{0}\left(\mathbb{P}^{r}, \Omega_{\mathbb{P}^{r}}^{1}(2)\right)
$$

Both these homomorphisms are isomorphisms, and therefore so is $\Phi_{H}$.

Lemma 7.2. Let $Y \subset \mathbb{P}^{r+1}$ for $r \geqslant 2$ be non-degenerate and linearly normal, and consider a hyperplane section $Z=H \cap Y \subset H \cong \mathbb{P}^{r}$. If $H^{1}\left(Z, \mathcal{I}_{Z / \mathbb{P}^{r}}^{2}(k)\right)=0$ for all $k$, then $H^{1}\left(Y, \mathcal{I}_{Y / \mathbb{P}^{r+1}}^{2}(k)\right)=0$ for all $k$.

Proof. We have exact sequences

$$
\begin{gathered}
0 \longrightarrow \mathcal{I}_{Y / \mathbb{P}^{r+1}}^{2}(k-1) \longrightarrow \mathcal{I}_{Y / \mathbb{P}^{r+1}}^{2}(k) \longrightarrow \mathcal{I}_{Z / \mathbb{P}^{r}}^{2}(k) \longrightarrow 0 \\
0 \longrightarrow \mathcal{I}_{Y / \mathbb{P}^{r+1}}^{2}(k) \longrightarrow \mathcal{I}_{Y / \mathbb{P}^{r+1}}(k) \longrightarrow N_{Y / \mathbb{P}^{r+1}}^{\vee}(k) \longrightarrow 0
\end{gathered}
$$

Since

$$
H^{0}\left(Y, N_{Y / \mathbb{P}^{r+1}}^{\vee}(k)\right)=0=H^{1}\left(\mathbb{P}^{r+1}, \mathcal{I}_{Y / \mathbb{P}^{r+1}}(k)\right)
$$

for $k \ll 0$, from $(7.4)$ we deduce that $H^{1}\left(\mathbb{P}^{r+1}, \mathcal{I}_{Y / \mathbb{P}^{r+1}}^{2}(k)\right)=0$ for $k \ll 0$. On the other hand, the hypothesis and (7.3) imply that

$$
H^{1}\left(\mathbb{P}^{r+1}, \mathcal{I}_{Y / \mathbb{P}^{r+1}}^{2}(k-1)\right) \longrightarrow H^{1}\left(\mathbb{P}^{r+1}, \mathcal{I}_{Y / \mathbb{P}^{r+1}}^{2}(k)\right)
$$

is a surjection for all $k$. This concludes the proof.

These two lemmas together imply the following.

Proposition 7.3. For all $k$, we have $H^{1}\left(\mathbb{P}, \mathcal{I}_{X / \mathbb{P}}^{2}(k)\right)=0$.

Proof. Recalling that $X$ is a cone over the smooth scroll $\mathbb{P}^{1} \times \mathbb{P}^{2} \subset \mathbb{P}^{5}$, the result is obtained by repeatedly applying Lemma 7.2 and using Lemma 7.1 at the first step.

In [Wah97], a similar result is proved for smooth scrolls. In our case, we cannot apply [Wah97, Proposition 4.6] directly.

\section{Proof of Theorem 1.3}

The proof will follow from an adaptation of [Wah97, Proposition 3.2] to our case.

Consider the following commutative diagram:

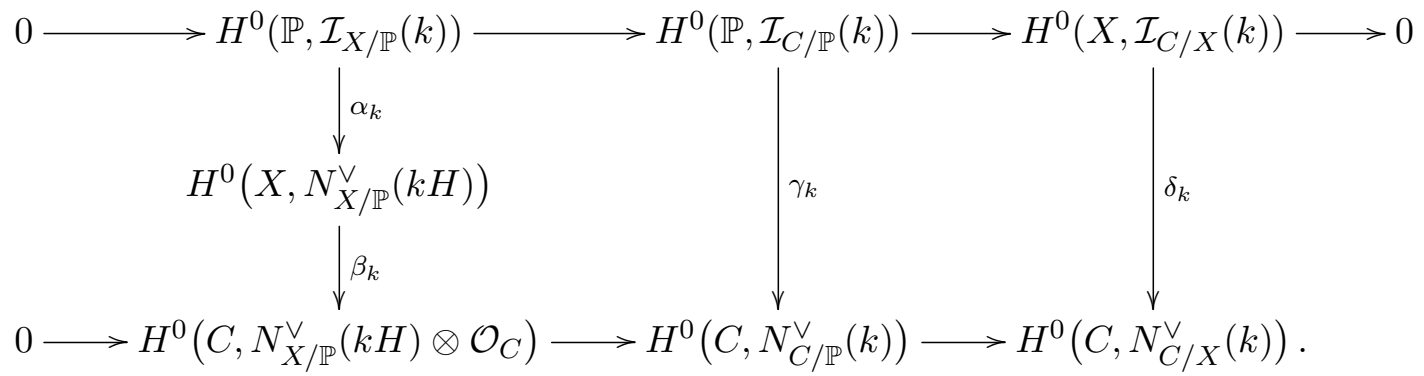




\section{ON HYPERPLANE SECTIONS OF K3 SURFACES}

Since $H^{1}\left(\mathbb{P}, \mathcal{I}_{C / \mathbb{P}}(k)\right)=0$ for $k \geqslant 3$, it will suffice to prove that $\gamma_{k}$ is surjective for all $k \geqslant 3$. From Propositions 6.1 and 7.3, we know that $\alpha_{k}$ and $\delta_{k}$ are surjective for $k \geqslant 3$. Therefore, it suffices to prove that $\beta_{k}$ is surjective for all $k \geqslant 3$.

Since $X$ is defined as the degeneracy locus of the matrix of the Petri map

$$
\mu_{0}(L): H^{0}(C, L) \otimes V \longrightarrow H^{0}\left(C, \omega_{C}\right),
$$

a free resolution of $\mathcal{I}_{X / \mathbb{P}}$ can be written under the form

$$
0 \longrightarrow H^{0}(C, L) \otimes \mathcal{O}_{\mathbb{P}}(-3) \stackrel{\psi}{\longrightarrow} V^{\vee} \otimes \mathcal{O}_{\mathbb{P}}(-2) \longrightarrow \mathcal{I}_{X / \mathbb{P}} \longrightarrow 0 .
$$

Restricting to $X$, we obtain

$$
0 \longrightarrow \mathcal{R} \longrightarrow H^{0}(C, L) \otimes \mathcal{O}_{X}(-3 H) \stackrel{\psi}{\longrightarrow} V^{\vee} \otimes \mathcal{O}_{X}(-2 H) \longrightarrow N_{X / \mathbb{P}}^{\vee} \longrightarrow 0,
$$

where neither $\mathcal{R}$ nor $N_{X / \mathbb{P}}^{\vee}$ is locally free because the rank of $\psi$ drops further on $\mathbb{P} W$.

Restricting to $C$, which is disjoint from $\mathbb{P} W$, we obtain from the base-point-free pencil trick an exact sequence as follows:

$$
0 \longrightarrow \omega_{C}^{-3} L^{-1} \longrightarrow H^{0}(C, L) \otimes \omega_{C}^{-3} \longrightarrow V^{\vee} \otimes \omega_{C}^{-2} \longrightarrow N_{X / \mathbb{P} \mid C}^{\vee} \longrightarrow 0 .
$$

From this sequence, we deduce the exact sequence

$$
0 \longrightarrow \omega_{C}^{-3} L \longrightarrow V^{\vee} \otimes \omega_{C}^{-2} \longrightarrow N_{X / \mathbb{P} \mid C}^{\vee} \longrightarrow 0 .
$$

Twisting this one by $\omega_{C}^{3}$, we obtain the exact sequence

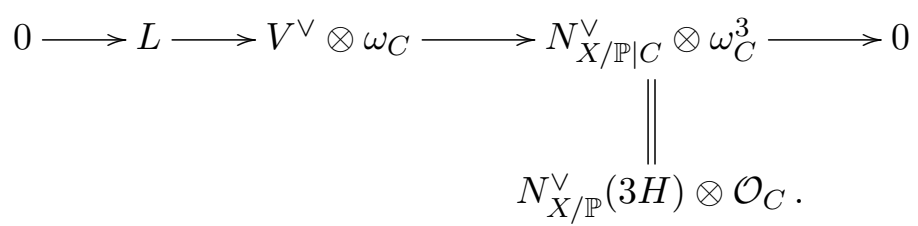

Since the induced homomorphism

$$
H^{1}(C, L) \longrightarrow V^{\vee} \otimes H^{1}\left(C, \omega_{C}\right)
$$

is the isomorphism given by Serre duality, we obtain that the homomorphism

$$
\phi: V^{\vee} \otimes H^{0}\left(C, \omega_{C}\right) \longrightarrow H^{0}\left(C, N_{X / \mathbb{P}}^{\vee}(3 H) \otimes \mathcal{O}_{C}\right)
$$

is surjective. Now, the diagram

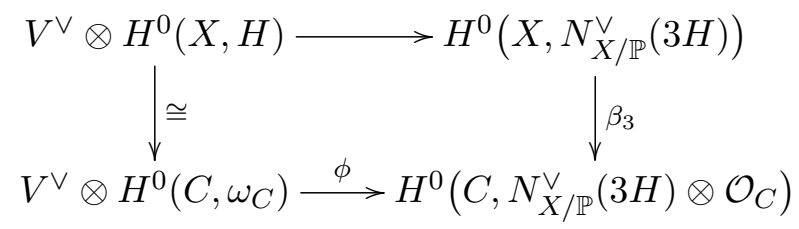

shows that $\beta_{3}$ is surjective. The surjectivity of $\beta_{k}$ for $k \geqslant 4$ is proved similarly.

\section{Part II.}

\section{Surfaces with canonical sections}

We follow the terminology and the notation of [Epe84, Epe83, CL02]. 


\section{E. Arbarello, A. Bruno and E. Sernesi}

Definition 9.1. A surface with canonical sections is a projective surface $\bar{S} \subset \mathbb{P}^{g}$, with $g \geqslant 3$, whose general section is a smooth canonical curve of genus $g$. We also require that $\bar{S}$ is not a cone.

From the definition, it follows that $\bar{S}$ is irreducible with at most isolated singularities. Consider the maps

$$
p: S \longrightarrow S_{0}, \quad q: S \longrightarrow \bar{S} \subset \mathbb{P}^{g},
$$

where $q$ is the minimal resolution and $S_{0}$ is a minimal model of $S$. The smooth surface $S$ is equipped with a big and nef divisor $C$, where $\left.\mathcal{O}_{S}(C)=q^{*} \mathcal{O}_{\bar{S}}(1)\right)$, with

$$
C^{2}=2 g-2, \quad K_{S} \otimes \mathcal{O}_{C}=\mathcal{O}_{C}, \quad h^{0}(S, C)=g+1 .
$$

In [Epe83, Epe84], Epema proves the following result (see also [Ume81] and Proposition 2.2 in [CL02]).

Proposition 9.2 ([Epe84]). Let $\bar{S} \subset \mathbb{P}^{g}$ be a surface with canonical sections. Then

(i) $\bar{S}$ is projectively normal,

(ii) $S$ is either a minimal K3 surface (possibly with rational singularities) or a ruled surface,

(iii) $h^{0}\left(S,-K_{S}\right)=1$.

If $\bar{S}$ is not a K3 surface, we have two possibilities for $S_{0}$ : either

$$
S_{0}=\mathbb{P}^{2} \quad \text { or } \quad S_{0}=\mathbb{P} E \stackrel{\pi}{\longrightarrow} \Gamma,
$$

where $\Gamma$ is a smooth curve of genus $\gamma$ and $E$ is a rank 2 vector bundle on $\Gamma$.

\subsection{Notation for the case $S_{0}=\mathbb{P}^{2}$}

From Proposition 9.2, there is a unique section $J \in H^{0}\left(S,-K_{S}\right)$. This curve is contracted to a point by $q$. We set

$$
C_{0}=p(C) \subset \mathbb{P}^{2}, \quad J_{0}=p(J) \subset \mathbb{P}^{2} .
$$

Then $C_{0}$ is an irreducible plane curve of degree $d$ and $J_{0}$ is a plane cubic passing through the singular points of $C_{0}$. We denote by the same symbol $\ell$ the class of a line in $\mathbb{P}^{2}$ and its pull-back, via $p$, to $S$. We have

$$
C \sim d \ell-\sum_{i=1}^{h} \nu_{i} E_{i}, \quad \nu_{1} \geqslant \nu_{2} \geqslant \cdots \geqslant \nu_{h} \geqslant 1,
$$

where $E_{i}$ is a chain of smooth rational curves, with $E_{i} \cdot E_{j}=-\delta_{i j}$. We set

$$
p_{i}=p\left(E_{i}\right), \quad i=1, \ldots, h,
$$

where $p_{1}, \ldots, p_{h}$ are (possibly infinitely near) points in $\mathbb{P}^{2}$. We have

$$
\begin{gathered}
K_{S} \sim-3 \ell+\sum_{i=1}^{h} E_{i}, \quad J \sim 3 \ell-\sum_{i=1}^{h} E_{i}, \quad K_{S} \cdot C=J \cdot C=0, \quad J^{2}=K_{S}^{2}=9-h, \\
|C|=\left\langle\left|K_{S}+C\right|+J, C\right\rangle, \quad \operatorname{dim}|C|=g, \quad \phi_{|C|}: S \rightarrow \bar{S} \subset \mathbb{P}^{g}
\end{gathered}
$$

(here $\langle A, B\rangle$ stands for the linear system spanned by $A$ and $B$ ). Finally, since $K_{S} \cdot C=0$, we have

$$
3 d=\sum_{i=1}^{h} \nu_{i}
$$




\section{ON HYPERPLANE SECTIONS OF K3 SURFACES}

so that

$$
2 g-2=d^{2}-\sum_{i=1}^{h} \nu_{i}^{2}
$$

Notice that $J^{2}<0$ implies $h \geqslant 10$.

\subsection{Notation for the case $\boldsymbol{S}_{\mathbf{0}}=\mathbb{P}(\boldsymbol{E})$}

In [Epe84, Proposition 1.4, p. 34], Epema proves that, after replacing $S_{0}$ with another minimal model if necessary, we can assume

$$
E=\mathcal{O}_{\Gamma} \oplus \mathcal{O}_{\Gamma}(D), \quad \operatorname{deg} D=-e,
$$

and furthermore $e>0$ (cf. Remark 9.4). We denote by $Z$ the unique member of $\left|-K_{S}\right|$. We have

$$
\begin{gathered}
Z \cdot C=0, \quad C^{2}=2 g-2, \\
|C|=\left\langle\left|K_{S}+C\right|+Z, C\right\rangle, \quad \operatorname{dim}|C|=g, \quad \phi_{|C|}: S \rightarrow \bar{S} \subset \mathbb{P}^{g} .
\end{gathered}
$$

We have the following standard situation:

$$
\operatorname{Pic}\left(S_{0}\right)=\sigma \mathbb{Z} \oplus \pi^{*} \operatorname{Pic}(\Gamma), \quad \sigma^{2}=-e, \quad \sigma \cdot \pi^{*}(x)=1, \quad x \in \Gamma .
$$

Also,

$$
K_{S_{0}} \sim-2 \sigma+\pi^{*}\left(K_{\Gamma}+D\right) .
$$

Let $C_{0}$ be the image of $C$ under the morphism $p: S \rightarrow S_{0}$. Then

$$
C_{0} \sim a\left(\sigma-\pi^{*}(D)\right)
$$

for some $a \geqslant 1$, so that

$$
C \sim p^{*}\left(C_{0}\right)-\sum_{i=1}^{h} \nu_{i} E_{i}=a p^{*}\left(\sigma-\pi^{*} D\right)-\sum_{i=1}^{h} \nu_{i} E_{i},
$$

where, as before, $E_{i}$ is a chain of smooth rational curves with $E_{i} \cdot E_{j}=-\delta_{i j}$, and where we assume

$$
\nu_{1} \geqslant \nu_{2} \geqslant \cdots \geqslant \nu_{h} \geqslant 1
$$

We also set

$$
p_{i}=p\left(E_{i}\right), \quad i=1, \ldots, h,
$$

where $p_{1}, \ldots, p_{h}$ are (possibly infinitely near) points in $\mathbb{P}(E)$. We then have

$$
\begin{aligned}
K_{S} & \sim p^{*}\left(K_{S_{0}}\right)+\sum_{i=1}^{h} E_{i}=p^{*}\left(-2 \sigma+\pi^{*}\left(K_{\Gamma}+D\right)\right)+\sum_{i=1}^{h} E_{i}, \\
Z & \sim p^{*}\left(2 \sigma-\pi^{*}\left(K_{\Gamma}+D\right)\right)-\sum_{i=1}^{h} E_{i} .
\end{aligned}
$$

Notice that since $p^{*} \sigma$ and $Z$ are effective divisors with $p^{*} \sigma \cdot Z<0$, we must have

$$
Z=p^{*} \sigma+J
$$

where $J$ is an effective divisor with

$$
J \sim p^{*}\left(\sigma-\pi^{*}\left(K_{\Gamma}+D\right)\right)-\sum_{i=1}^{h} E_{i} .
$$




\section{E. Arbarello, A. Bruno and E. Sernesi}

Notice that in the case $\gamma=1$,

$$
p^{*}(\sigma)^{2}=-e, \quad J^{2}=e-h, \quad p^{*}(\sigma) \cdot J=0 .
$$

In the case $\gamma=1$, we have, more precisely, $-D=\sum_{i=1}^{e} x_{i}$ and $\sigma-\pi^{*}\left(K_{\Gamma}+D\right)=\sigma+\sum f_{i}$, where $f_{i}=\pi^{-1}\left(x_{i}\right)$. All the members of the anti-canonical system $\left|K_{S_{0}}\right|$ are reducible and of the form $\sigma+J_{0}$ with $J_{0} \in\left|\sigma+\sum f_{i}\right|$. Moreover, $\operatorname{dim}\left(\left|\sigma+\sum_{i} f_{i}\right|\right)=e$, as follows from the exact sequence on $S_{0}$

$$
0 \longrightarrow \mathcal{O}\left(\sum f_{i}\right) \longrightarrow \mathcal{O}\left(\sigma+\sum f_{i}\right) \longrightarrow \mathcal{O}_{\sigma} \longrightarrow 0
$$

and from $H^{1}\left(\mathcal{O}\left(\sum f_{i}\right)\right)=0$. An analogous exact sequence shows that for each effective divisor $\sum_{k=1}^{e-1} y_{k}$ on $\Gamma$ of degree $e-1$, we have $\operatorname{dim}\left(\left|\sigma+\sum \pi^{-1}\left(y_{k}\right)\right|\right)=e-2$. Therefore, the general member of $\left|\sigma+\sum_{i} f_{i}\right|$ is irreducible, and if $J_{0} \in\left|\sigma+\sum_{i} f_{i}\right|$ contains a fibre of $\pi$ as a component, then it splits as $J_{0}=\sigma+\sum \pi^{-1}\left(y_{i}\right)$ for some $\sum y_{i} \in|D|$. Accordingly, $J \subset S$ either is irreducible or splits as $p^{*} \sigma+\sum \varphi_{i}$, where the $\varphi_{i}$ are the proper transforms of fibres of $\pi$.

Epema's Proposition 1.4 in [Epe84] (see also [CL02, Proposition 2.6]), contains the following facts and some more; see, for instance, [Epe84, Chapter IV] for the slight modifications in case $\gamma=1$.

Proposition 9.3 ([Epe84]). Suppose $S_{0}=\mathbb{P}(E)$. After replacing $S_{0}$ with another minimal model we can assume that the following hold:

(i) $E=\mathcal{O}_{\Gamma} \oplus \mathcal{O}_{\Gamma}(D), \operatorname{deg} D=-e$.

(ii) Either $D \sim-K_{\Gamma}$, or $h \geqslant 1$ and $e>2 \gamma-2$.

(iii) We have $\nu_{i} \leqslant a-1$ for $i=1, \ldots, h$.

(iv) $\sum_{i=1}^{h} \nu_{i}=a(e-2 \gamma+2)$.

(v) $2 g-2=a^{2} e-\sum_{i=1}^{h} \nu_{i}^{2}$.

(vi) $2 a^{2}(\gamma-1)+a(e-2 \gamma+2) \leqslant 2 g-2 \leqslant a^{2} e-a(e-2 \gamma+2)$.

(vii) We have $(a-1) h \leqslant 2\left[g-1-a^{2}(\gamma-1)\right]$ for $a \geqslant 2$.

(viii) None of the base points of $\left|C_{0}\right|$ lie on $\sigma \subset S_{0}$.

By virtue of part (viii), we will often use the same symbol $\sigma$ to denote the minimal section $\sigma \subset S_{0}$ and its pull-back $p^{*} \sigma \subset S$ under the minimal model map $p: S \rightarrow S_{0}$.

Remark 9.4. Observe that if $g \geqslant 2$, the genus formula (v) gives $e \geqslant 1$. We also have $a \geqslant 2$, by part (iii).

\section{Smoothing}

Let $g \geqslant 3$ and consider again the situation of (9.1):

$$
p: S \longrightarrow S_{0}, \quad q: S \longrightarrow \bar{S} \subset \mathbb{P}^{g},
$$

We keep the same notation as in Section 9.

Lemma 10.1. Let $T_{\bar{S}}=\operatorname{Hom}\left(\Omega_{\bar{S}}, \mathcal{O}_{\bar{S}}\right)$; then $H^{2}\left(\bar{S}, T_{\bar{S}}\right)=0$.

Proof. We have $T_{\bar{S}}=q_{*} T_{S}$ (Proposition 9.2 and [BW74, Proposition 1.2]) and therefore

$$
H^{2}\left(\bar{S}, T_{\bar{S}}\right)=H^{2}\left(S, T_{S}\right)
$$




\section{ON HYPERPLANE SECTIONS OF K3 SURFACES}

Since $p$ is a sequence of blow-ups at non-singular points, we also have [Ser06, Example 2.4.11(iii)]

$$
H^{2}\left(S, T_{S}\right)=H^{2}\left(S_{0}, T_{S_{0}}\right)=0 .
$$

Let $L=\mathcal{O}_{\bar{S}}(1)$, and let $c(L) \in H^{1}\left(\bar{S}, \Omega \frac{1}{\bar{S}}\right)$ be its Chern-Atiyah class. It corresponds to an extension

$$
0 \rightarrow \Omega \frac{1}{S} \rightarrow \mathcal{Q}_{L} \rightarrow \mathcal{O}_{\bar{S}} \rightarrow 0
$$

Set $\mathcal{E}_{L}=\mathcal{Q}_{L}^{\vee}$, and consider the dual exact sequence

$$
0 \rightarrow \mathcal{O}_{\bar{S}} \rightarrow \mathcal{E}_{L} \rightarrow T_{\bar{S}} \rightarrow 0 .
$$

LEMma 10.2. $H^{2}\left(\bar{S}, \mathcal{E}_{L}\right)=0$.

Proof. In all cases, $\bar{S}$ has Gorenstein normal singularities since $\bar{S}$ is projectively normal, $\omega_{S}=$ $\mathcal{O}_{S}(-Z)$ and $Z$ is supported on the exceptional locus of $q$. Moreover, $\omega_{\bar{S}}=\mathcal{O}_{\bar{S}}$ by adjunction. Since by Serre duality,

$$
H^{2}\left(\bar{S}, \mathcal{O}_{\bar{S}}\right)=H^{2}\left(\bar{S}, \omega_{\bar{S}}\right) \cong H^{0}\left(\bar{S}, \mathcal{O}_{\bar{S}}\right)^{\vee} \cong \mathbb{C},
$$

it suffices to prove that the coboundary map

$$
\partial: H^{1}\left(\bar{S}, T_{\bar{S}}\right) \rightarrow H^{2}\left(\bar{S}, \mathcal{O}_{\bar{S}}\right)
$$

from the exact sequence (10.2) is non-zero. The map $\partial$ is the cup product with the extension class defining (10.2). This class is a non-zero element of $\operatorname{Ext}^{1}\left(T_{\bar{S}}, \mathcal{O}_{\bar{S}}\right)$, and this space is the Serre dual of $H^{1}\left(\bar{S}, T_{\bar{S}}\right)$. Hence $\partial$ is non-zero.

Consider the deformation functor $\operatorname{Def}_{(\bar{S}, L)}$ of (not necessarily locally trivial) deformations of the pair $(\bar{S}, L)$. In $[\mathrm{AC} 10]$, it is shown that

$$
\operatorname{Def}_{(\bar{S}, L)}(\mathbb{C}[\epsilon])=\operatorname{Ext}^{1}\left(\mathcal{Q}_{L}, \mathcal{O}_{\bar{S}}\right)
$$

and that the obstruction space is contained in $\operatorname{Ext}^{2}\left(\mathcal{Q}_{L}, \mathcal{O}_{\bar{S}}\right)$.

We have a morphism of functors

$$
\mu: \operatorname{Def}_{(\bar{S}, L)} \longrightarrow \coprod_{\bar{s} \in \operatorname{Sing}(\bar{S})} \operatorname{Def}_{\mathcal{O}_{\bar{S}, \bar{s}}}
$$

defined by restriction.

TheOrem 10.3. The morphism $\mu$ is smooth. In particular, if $\bar{S}$ has smoothable singularities then $[\bar{S}] \in$ Hilb $^{\mathbb{P}^{g}}$ belongs to the closure of the family of non-singular K3 surfaces of degree $2 g-2$.

Proof. The exact sequence coming from the local-to-global spectral sequence for Ext implies the following exact sequence (see [AC10, Theorem 3.1]):

$$
0 \rightarrow H^{1}\left(\bar{S}, \mathcal{E}_{L}\right) \rightarrow \operatorname{Ext}^{1}\left(\mathcal{Q}_{L}, \mathcal{O}_{\bar{S}}\right) \stackrel{d \mu}{\longrightarrow} H^{0}\left(\bar{S}, T_{\bar{S}}^{1}\right) \rightarrow H^{2}\left(\bar{S}, \mathcal{E}_{L}\right)
$$

where $d \mu$ is the differential of $\mu$. From Lemma 10.2, we obtain that $d \mu$ is surjective.

Lemma 10.2 and the fact that $\mathcal{E x t}^{1}\left(\mathcal{Q}_{L}, \mathcal{O}_{\bar{S}}\right)$ has finite support imply that

$$
\operatorname{Ext}^{2}\left(\mathcal{Q}_{L}, \mathcal{O}_{\bar{S}}\right)=H^{0}\left(\mathcal{E x t}^{2}\left(\mathcal{Q}_{L}, \mathcal{O}_{\bar{S}}\right)\right)
$$

Moreover, since $\bar{S}$ is normal, we have [Ser06, Proposition 3.1.14]

$$
\mathcal{E x t}^{2}\left(\Omega \frac{1}{\bar{S}}, \mathcal{O}_{\bar{S}}\right) \cong T_{\bar{S}}^{2} .
$$




\section{E. Arbarello, A. Bruno and E. Sernesi}

From the exact sequence (10.1), we deduce that

$$
\mathcal{E}_{\mathrm{xt}^{2}}\left(\mathcal{Q}_{L}, \mathcal{O}_{\bar{S}}\right) \subset \mathcal{E}_{\mathrm{xt}^{2}}\left(\Omega_{\bar{S}}^{1}, \mathcal{O}_{\bar{S}}\right)
$$

Therefore, the obstruction map

$$
o(\mu): H^{0}\left(\mathcal{E}_{\mathrm{xt}^{2}}\left(\mathcal{Q}_{L}, \mathcal{O}_{\bar{S}}\right)\right) \rightarrow H^{0}\left(\bar{S}, T_{\bar{S}}^{2}\right)
$$

is injective. Therefore, $\operatorname{Def}_{(\bar{S}, L)}$ is less obstructed than $\coprod_{\bar{s} \in \operatorname{Sing}(\bar{S})} \operatorname{Def}_{\mathcal{O}_{\bar{S}, \bar{s}}}$, and this implies that $\mu$ is smooth.

Since the singularities of $\bar{S}$ are smoothable, from the smoothness of $\mu$ it follows that the pair $(\bar{S}, L)$ deforms to a non-singular pair $\left(X, \mathcal{O}_{X}(1)\right)$. Moreover, the fact that $H^{1}(L)=0$ implies that all the sections of $L$ extend and the theorem follows.

COROllary 10.4. With the same notation as before, assume that we are in one of the following cases:

(i) We have $S_{0}=\mathbb{P}^{2}$ and $K_{S}^{2}=9-h$.

(ii) We have $S_{0}=\mathbb{P}(E)$, where $E=\mathcal{O}_{\Gamma} \oplus \mathcal{O}_{\Gamma}(D)$ with $\Gamma$ a smooth curve of genus $1, \operatorname{deg}(D)=-e$ and $K_{S}^{2}=-h$ with $h \geqslant e$.

Then $\bar{S} \subset \mathbb{P}^{g}$ deforms to a surface $X \subset \mathbb{P}^{g}$ such that

Case (i): the surface $X$ has a simple elliptic singularity of degree $h-9$ and its minimal resolution is the blow-up of $h$ distinct points on a non-singular cubic in $\mathbb{P}^{2}$;

Case (ii): the surface $X$ has two simple elliptic singularities of degrees $e$ and $h-e$, respectively.

Proof. (i) From [Ume81, Theorem 1], it follows that the singularity $\bar{s} \in \bar{S}$ satisfies $p_{g}(\bar{s})=1$, and therefore it is a minimally elliptic singularity [Lau77, Theorem 3.10]. This singularity, being obtained by blowing up $h$ (possibly infinitely near) points on a plane cubic $J_{0}$, can be deformed to a simple elliptic singularity obtained by making $J_{0}$ non-singular and the $h$ points distinct. This deformation can be obtained by deforming $\bar{S}$ in $\mathbb{P}^{g}$, by Theorem 10.3.

(ii) Theorem 1 of [Ume81] implies that $\bar{S}$ has either two simple elliptic singularities or one normal Gorenstein singularity of geometric genus 2. In the first case, there is nothing to prove. The second case arises when the component $J$ of $Z$ (cf. (9.6), (9.7)) splits as $p^{*} \sigma+\sum \varphi_{i}$, where the $\varphi_{i}$ are the proper transforms of fibres of $\pi$. Since $h \geqslant e$, we can move the points $p_{1}, \ldots, p_{h}$ so that they do not belong to a reducible curve of the form $\sum \pi^{-1}\left(y_{i}\right)$, for some $\sum y_{i} \in|D|$, and thus make $J$ irreducible. This means that the second case is a degeneration of the first one obtained by moving the points $p_{1}, \ldots, p_{h} \in S_{0}$ so as to make $J$ reducible. Theorem 10.3 implies that this deformation can be realised by deforming $\bar{S}$ in $\mathbb{P}^{g}$.

Corollary 10.5. In the same situation of Corollary 10.4, assume further the following:

Case (i): $-9 \leqslant K_{S}^{2} \leqslant-1$.

Case (ii): $1 \leqslant e \leqslant h-1$ and $2 \leqslant h \leqslant 10$.

Then $\bar{S}$ deforms in $\mathbb{P}^{g}$ to a non-singular K3 surface of degree $2 g-2$.

Proof. Corollary 10.4 implies that $\bar{S}$ deforms to a surface $X \subset \mathbb{P}^{g}$ having only simple elliptic singularities. Such singularities are analytically equivalent to a cone $C_{E}$ over an elliptic curve $E \subset \mathbb{P}^{k-1}$ embedded with degree $k$ if $k \geqslant 3$ or to a hypersurface singularity if $k=1,2$ [Lau77, Theorem 3.13]. In the cases considered, $k=-K_{S}^{2}$ in case (i), and $k=e$ and $k=h-e$ in case (ii). In all such cases (and in the cases $k=1,2$ ), the singularities of $X$ are smoothable (see [Pin74]). Therefore, the corollary follows by applying Theorem 10.3 to $X$. 


\section{ON HYPERPLANE SECTIONS OF K3 SURFACES}

Remark 10.6. Theorem 10.3 is related to results of Karras, Looijenga, Pinkham, Wahl [Kar77, Loo81, Wah81, Pin77] about the possibility of inducing all deformations of a normal (elliptic) singularity of a compact surface by means of global abstract deformations of the surface. Our result involves a polarisation in its statement, assuring that, in the case of surfaces with canonical sections in $\mathbb{P}^{g}$, the deformations of the singularity can be realised by deforming the surface in $\mathbb{P}^{g}$. At the first-order level, one can compare the two cases (abstract vs embedded deformations) by means of the following diagram:

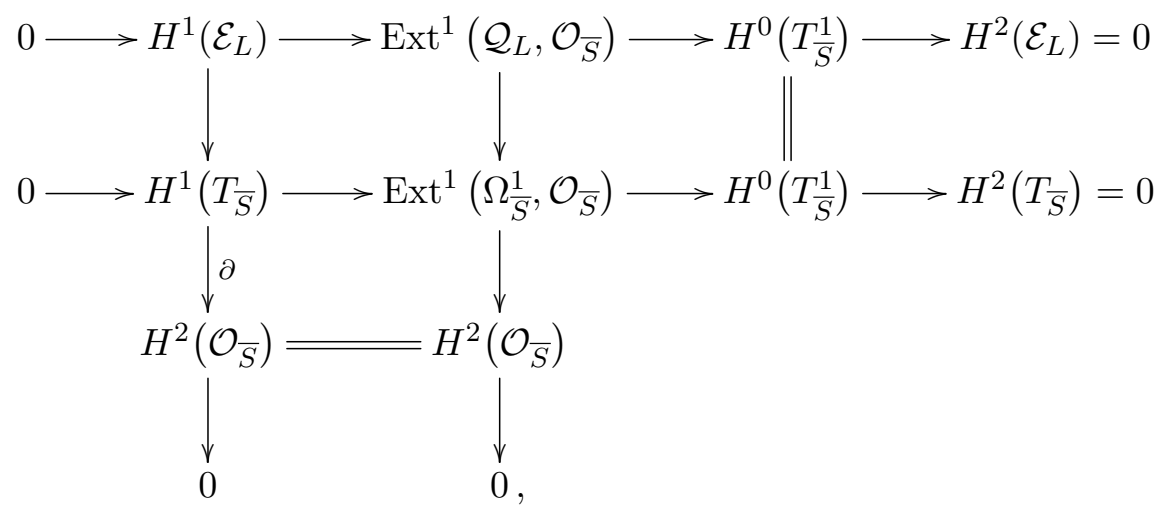

where the horizontal sequences are the local-global sequences of the Ext functors.

More recently, smoothing techniques similar to ours have been applied to the construction of surfaces of general type (see [KLP12] and references therein).

\section{The main theorem for BNP curves on surfaces with canonical sections}

Our main theorem for BNP curves on surfaces with canonical sections is the following.

Theorem 11.1. Let $C$ be a Brill-Noether-Petri curve of genus $g \geqslant 12$. Then $C$ lies on a polarised K3 surface or on a limit thereof if and only if the Wahl map is not surjective.

Let us go back to diagram (9.1) and consider the curve $C_{0} \subset S_{0}$. In the next three sections, we will analyse the restrictions imposed on the singularities of $C_{0}$ when we assume that $C$ is a BNP curve. We first study the case in which $S_{0}=\mathbb{P}(E) \rightarrow \Gamma$ is a ruled surface with $g(\Gamma)=\gamma$, and in Sections 11.1, 11.2 and 11.3, we will address the cases in which $\gamma>1, \gamma=1$ and $\gamma=0$, respectively. In Section 11.4, we will study the case $S_{0}=\mathbb{P}^{2}$.

Referring to the notation we established in Sections 9.1 and 9.2, we will prove the following proposition.

Proposition 11.2. Let $\bar{S} \subset \mathbb{P}^{g}$ be a surface with canonical sections, and let $C$ be a general hyperplane section of $\bar{S}$. Suppose that $C$ is a BNP curve.

(i) Suppose $S_{0}=\mathbb{P}(E)$, with $E$ a rank 2 vector bundle over a smooth curve $\Gamma$ of genus $\gamma$. Assume $g \geqslant 12$; then $\gamma \leqslant 1$.

(ii) Suppose $S_{0}=\mathbb{P}(E)$, with $E$ a rank 2 vector bundle over an elliptic curve $\Gamma$. Assume $g \geqslant 12$; then $e+1 \leqslant h \leqslant 7$ (recall that $\sigma^{2}=-e$ and $J^{2}=e-h$ ).

(iii) The case $S_{0}=\mathbb{P}(E)$, with $E$ a rank 2 vector bundle over a smooth rational curve $\Gamma$, can be reduced to next one.

(iv) Suppose $S_{0}=\mathbb{P}^{2}$, and assume $g \geqslant 12$. Then $10 \leqslant h \leqslant 18$, and therefore $K_{S}^{2}=9-h \geqslant-9$. 


\section{E. Arbarello, A. Bruno and E. Sernesi}

Let us assume Proposition 11.2, and let us prove the main theorem.

Proof of Theorem 11.1. Because of Wahl's theorem [Wah87] (see also Beauville and Merindol's proof [BM87]), there is only one implication to be proved. Let $C$ be a Brill-Noether-Petri curve of genus $g \geqslant 12$, for which the Wahl map is not surjective. From Theorem 1.3 and from Wahl's Theorem 1.2, we conclude that $C$ is extendable. Let $\bar{S} \subset \mathbb{P}^{g}$ be a surface with canonical sections having $C$ as one of its hyperplane sections. If $\bar{S}$ is not already a K3 surface, we are in the situation of diagram (9.1). By Corollary 10.4, we may assume that the anti-canonical divisor of the surface $S$ is smooth (with one or two connected components). By Proposition 11.2, we are either in case (ii) or in case (iv) and, moreover, each component of the anti-canonical divisor of $S$ has self-intersection at least -9 . It now suffices to apply Corollary 10.5 to deduce that $\bar{S}$ is a limit of K3 surfaces.

\subsection{Ruled surfaces of genus $\gamma>1$}

In this section, the surfaces $S$ and $S_{0}=\mathbb{P}(E)$ and the curve $C$ will be as described in Section 9.2. The next proposition gives Proposition 11.2(i).

Proposition 11.3. Assume that $C$ is a BNP curve of genus $g \geqslant 12$. Then $\gamma \leqslant 1$ and $a \geqslant 3$.

Lemma 11.4. Suppose that $C$ is a BNP curve. Then

$$
a(\gamma+3) \geqslant g+2 .
$$

Proof. Consider a $g_{k}^{1}$ on $\Gamma$, and lift it to $C$ via the composition $\pi \cdot p: C \rightarrow \Gamma$. This is a degree $a$ morphism. We then get a pencil of degree $a k$ on $C$. As $C$ is BNP, we must have $2 a k \geqslant g+2$. If the $g_{k}^{1}$ on $\Gamma$ is of minimal degree, we have $2 k \leqslant \gamma+3$, so that $a(\gamma+3) \geqslant 2 a k \geqslant g+2$.

Lemma 11.5. If $C$ is a BNP curve, we have $(a-2)(\gamma-1)+e / 2 \leqslant 4-3 / a$.

Proof. The first inequality in Proposition 9.3(vi) can be written as

$$
1+\left(a^{2}-a\right)(\gamma-1)+\frac{a e}{2} \leqslant g .
$$

This, together with (11.1), gives

$$
1+\left(a^{2}-a\right)(\gamma-1)+\frac{a e}{2} \leqslant g \leqslant a(\gamma+3)-2=a(\gamma-1)+4 a-2,
$$

and therefore

$$
\left(a^{2}-2 a\right)(\gamma-1)+\frac{a e}{2} \leqslant 4 a-3 .
$$

Dividing by $a$, we get the lemma.

Proof of Proposition 11.3. Assume $a=2$. From Lemma 11.5 , we get $e / 2 \leqslant 4-3 / a=5 / 2$, so that $e \leqslant 5$. From Proposition 9.3(v), we then get $g \leqslant 1+2 e \leqslant 11$, which is excluded by hypothesis. We may then assume $a \geqslant 3$. Since $2 \gamma-2 \leqslant e$, Lemma 11.5 gives

$$
2(\gamma-1) \leqslant(a-1)(\gamma-1)<4
$$

so that $\gamma \leqslant 2$. Suppose $\gamma=2$; then $a \leqslant 4$. If $a=4$, from Lemma 11.5 we get $e \leqslant 2$, so that $e=2$. From Proposition 9.3(iv), we deduce that $C_{0}$ is smooth and $g=17$. But then since $\Gamma$ is hyperelliptic, there is a $g_{8}^{1}$ on $C$ with negative Brill-Noether number, contrary to the assumption. In conclusion, if $\gamma=2$, we must have $a=3$. Pulling back on $C$ the $g_{2}^{1}$ on $\Gamma$, the BNP property yields $2 a \geqslant(g+2) / 2$, giving $g \leqslant 10$. 


\section{ON HYPERPLANE SECTIONS OF K3 SURFACES}

\subsection{Elliptic ruled surfaces}

In this section, our aim is to prove Proposition 11.2(ii). When $\gamma=1$, the setting is the following. The minimal model $S_{0}$ of $S$ is a ruled surface

$$
\pi: S_{0}=\mathbb{P} E \rightarrow \Gamma
$$

over an elliptic curve $\Gamma$ and $E=\mathcal{O}_{\Gamma} \oplus \mathcal{O}_{\Gamma}(D)$. Also,

$$
\begin{gathered}
K_{S_{0}} \sim-2 \sigma+\pi^{*} D, \quad \operatorname{deg} D=-e, \quad K_{S_{0}}^{2}=0, \\
K_{S} \sim p^{*}\left(-2 \sigma+\pi^{*} D\right)+\sum_{i=1}^{h} E_{i}, \quad K_{S}^{2}=-h,
\end{gathered}
$$

where $\sigma \subset S_{0}$ is a minimal section and $p: S \rightarrow S_{0}$ is the minimal model map. The anti-canonical divisor $Z \in\left|-K_{S}\right|$ is given by

$$
Z \sim p^{*} \sigma+J, \quad \text { where } \quad J \sim p^{*}\left(\sigma-\pi^{*} D\right)-\sum_{i=1}^{h} E_{i},
$$

and $p^{*} \sigma$ and $J$ are effective divisors such that

$$
\left(p^{*} \sigma\right)^{2}=\sigma^{2}=-e, \quad J^{2}=e-h, \quad p^{*} \sigma \cdot J=0 .
$$

The curve $C \subset S$ is given by

$$
C \sim a p^{*}\left(\sigma-\pi^{*} D\right)-\sum_{i=1}^{h} \nu_{i} E_{i}, \quad \nu_{1} \geqslant \cdots \geqslant \nu_{h} \geqslant 1, \quad a \geqslant 3, \quad e \geqslant 1 .
$$

The following proposition gives Proposition 11.2(ii).

Proposition 11.6. Suppose that $C$ is a BNP curve of genus $g \geqslant 12$. Then $e+1 \leqslant h \leqslant 7$.

The probe we will often use to test the BNP property of $C$ is the pencil on $S$ given by

$$
|M|=\left|p^{*}\left(\sigma-\pi^{*} D\right)-E_{1}-\cdots-E_{e-1}\right| .
$$

The fact that, indeed, this is at least a pencil follows from the fact that

$$
\begin{aligned}
h^{0}\left(S, p^{*}\left(\sigma-\pi^{*} D\right)\right) & =h^{0}\left(S_{0}, \sigma-\pi^{*} D\right)=h^{0}\left(\Gamma, \pi_{*}\left(\sigma-\pi^{*} D\right)\right) \\
& =h^{0}\left(\Gamma,\left(\mathcal{O}_{\Gamma} \oplus \mathcal{O}_{\Gamma}(D)\right) \otimes \mathcal{O}_{\Gamma}(-D)\right)=e+1
\end{aligned}
$$

and from Proposition 9.3(ii). Set $M_{C}=M \otimes \mathcal{O}_{C}$. Consider the exact sequence

$$
0 \rightarrow \mathcal{O}_{S}(M-C) \rightarrow \mathcal{O}_{S}(M) \rightarrow \mathcal{O}_{C}\left(M_{C}\right) \rightarrow 0 .
$$

By restricting to $\pi^{*}(x)$, we see that $h^{0}(S, M-C)=0$, so that the linear series $\left|M_{C}\right|$ is at least a pencil. A way to enforce the BNP property of $C$ will be to insist that

$$
h^{0}\left(C, K M_{C}^{-2}\right)=0 \text {. }
$$

Looking at the exact sequence

$$
0 \longrightarrow \mathcal{O}_{S}(-2 M) \longrightarrow \mathcal{O}_{S}(C-2 M) \longrightarrow \mathcal{O}_{C}\left(K M_{C}^{-2}\right) \longrightarrow 0
$$

and noticing that $h^{0}(S,-2 M)=0$, we see that a necessary condition for (11.5) to hold is that

$$
h^{0}(S, C-2 M)=0
$$

holds. Hence if $C$ is a BNP curve, (11.6) must hold. We come to the first lemma. 


\section{E. Arbarello, A. Bruno and E. Sernesi}

Lemma 11.7. Suppose that $C$ is a BNP curve, and let $g>4$. Then

(i) $4 a \geqslant g+4$,

(ii) $\nu_{e}=a-1$.

Proof. The elliptic curve $\Gamma$ parametrises infinitely many $g_{2}^{1}$ on itself. As a consequence, $W_{2 a}^{1}(C)$ contains an elliptic curve. Since $W_{2 a}^{1}(C)$ is connected and $C$ is BNP curve, by the genus formula for $W_{d}^{1}$ when $\rho=1$ (see, for example, [Ort13, p. 811]), we must have

$$
2 \leqslant \operatorname{dim} W_{2 a}^{1}(C)=\rho(g, 2 a, 1)=4 a-g-2
$$

proving statement (i). Let us prove statement (ii). Suppose that it does not hold. From Proposition 9.3(iii), we then get $\nu_{e} \leqslant a-2$. As a consequence, we have

$$
\begin{aligned}
\nu_{1}, \ldots, \nu_{e-1} & \leqslant a-1, \\
\nu_{j} & \leqslant a-2, \quad j=e, \ldots, h .
\end{aligned}
$$

This means that

$$
\begin{gathered}
(a-2)-\left(\nu_{i}-1\right) \geqslant 0, \quad i \leqslant e-1, \\
(a-2)-\nu_{j} \geqslant 0, \quad j \geqslant e .
\end{gathered}
$$

Now, consider the pencil $|M|$ defined in (11.3). Using (11.7), we get

$$
\begin{aligned}
C-2 M & =(a-2) p^{*}\left(\sigma-\pi^{*} D\right)-\sum_{i=1}^{e-1}\left(\nu_{i}-2\right) E_{i}-\sum_{i=e}^{h} \nu_{i} E_{i} \\
& =(a-2) J+\sum_{i=1}^{e-1}\left[(a-2)-\left(\nu_{i}-2\right)\right] E_{i}+\sum_{i=e}^{h}\left[(a-2)-\nu_{i} E_{i}\right],
\end{aligned}
$$

where $J$ is defined in (11.2). But then $C-2 M$ is an effective divisor, contrary to assumption (11.6).

Lemma 11.7 and Proposition 9.3(iii), (iv) and (v), put us in the following situation:

$$
\begin{gathered}
4 a \geqslant g+4, \quad \nu_{1}=\cdots=\nu_{e}=a-1 \geqslant \nu_{e+1} \geqslant \cdots \geqslant \nu_{h} \geqslant 1, \\
2 g-2=e(2 a-1)-\sum_{i=e+1}^{h} \nu_{i}^{2}, \quad e=\sum_{i=e+1}^{h} \nu_{i} .
\end{gathered}
$$

Proof of Proposition 11.6. From Proposition 9.3(vii) and the first inequality in (11.2), we get $h \leqslant 7$. In particular, $e+1 \leqslant h \leqslant 7$.

\subsection{Rational ruled surfaces}

In this section, we will assume $\gamma=0$. Our aim is to prove Proposition 11.2(iii). The setting is the following: the minimal model of $S$ is the rational ruled surface $S_{0}=\mathbb{F}_{e}$, that is,

$$
\pi: S_{0}=\mathbb{F}_{e}=\mathbb{P}(E) \rightarrow \Gamma,
$$

where $E=\mathcal{O}_{\Gamma} \oplus \mathcal{O}_{\Gamma}(-e)$. We recall that

$$
K_{\mathbb{F}_{e}} \sim-2 \sigma-(e+2) f,
$$

where $f$ is the class of a fibre and that

$$
K_{S} \sim p^{*}(-2 \sigma-(e+2) f)+\sum E_{i},
$$




\section{ON HYPERPLANE SECTIONS OF K3 SURFACES}

where $\sigma \subset \mathbb{F}_{e}$ is a minimal section with $\sigma^{2}=-e$. From Proposition 9.3, there exists a unique divisor

$$
Z \in H^{0}\left(S,-K_{S}\right)
$$

such that $Z \cdot C=0$. Furthermore, the class of $C \subset S$ is

$$
C=p^{*}(a \sigma+a e f)-\sum_{i=1}^{h} \nu_{i} E_{i} .
$$

It is convenient to write $\left(S_{e}, C_{e}, Z_{e}, p_{e}, S_{0, e}, C_{0, e}, Z_{0, e}, \sigma_{e}\right)$ instead of $\left(S, C, Z, p, S_{0}, C_{0}, Z_{0}, \sigma\right)$. Choose a point

$$
x_{e} \in Z_{e}, \quad x_{e} \notin C_{e} \cup \sigma_{e} .
$$

Let us perform the elementary transformation of $S_{e}$ centred at $x_{e}$ (see [Dol12, Section 7.4]): We first blow up $S_{e}$ at $x_{e}$ :

$$
s_{e}: X_{e} \longrightarrow S_{e}
$$

with exceptional divisor $E_{x_{e}} \subset X_{e}$. Since the strict transform $F_{x_{e}}=s^{*} f_{x_{e}}-E_{x_{e}}$ is an exceptional curve of the first kind, we can contract it. The blow-down of $F_{x_{e}}$ is a morphism

$$
t_{e-1}: X_{e} \longrightarrow S_{e-1},
$$

where $S_{e-1}$ is a non-minimal ruled surface, with a section $\sigma_{e-1}:=t_{e-1}\left(s_{e}^{-1}\right)\left(\sigma_{e}\right)$ of self-intersection

$$
\sigma_{e-1}^{2}=\left(t_{e-1}^{*}\left(\sigma_{e-1}\right)\right)^{2}=\left(s_{e}^{*}\left(\sigma_{e}\right)+F_{x_{e}}\right)^{2}=-e+1 .
$$

By construction, it follows that if

$$
p_{e-1}: S_{e-1} \longrightarrow S_{0, e-1}
$$

is the blow down of $E_{1}, \ldots, E_{h}$, then $S_{0, e-1}$ is the minimal ruled surface $\mathbb{F}_{e-1}$. Summarising the construction, we have a diagram

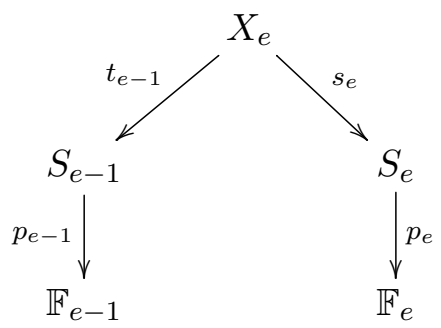

in which $F_{x_{e}}$ is $\left(t_{e-1}\right)$-exceptional and $E_{x_{e}}$ is $\left(s_{e}\right)$-exceptional. If, by a slight abuse of notation, we denote by $f$ a general fibre in both $S_{e}$ and $S_{e-1}$, we have on $X_{e}$ the linear equivalence

$$
s_{e}^{*} f=t_{e-1}^{*} f \sim E_{x_{e}}+F_{x_{e}} .
$$

Let us denote by $\bar{D}$ the strict transform of a divisor $D$ under $s_{e}$ or $t_{e-1}$. By construction, we have

$$
s_{e}^{*} C_{e}=\bar{C}_{e}, \quad s_{e}^{*} \sigma_{e}=\bar{\sigma}_{e}=t_{e-1}^{*} \sigma_{e-1}-F_{x_{e}} .
$$

Consider the image under $t_{e-1}$ of the strict transform of $C_{e}$, and set

$$
C_{e-1}:=t_{e-1}\left(\bar{C}_{e}\right) \text {. }
$$

This is an irreducible curve birational to $C_{e}$, which has a point of multiplicity $a$ at the point 


\section{E. Arbarello, A. Bruno and E. Sernesi}

$t_{e-1}\left(F_{x_{e}}\right)=C_{e-1} \cap \sigma_{e-1}$. Let us also consider the projection of $C_{e-1}$ to $\mathbb{F}_{e-1}$

$$
C_{0, e-1}:=p_{e-1}\left(C_{e-1}\right) \text {. }
$$

The class of $C_{0, e-1} \subset \mathbb{F}_{e-1}$ is $a \sigma_{e-1}+e a f$, because $\left.t_{e-1}^{*}\left(p_{e-1}^{*}\left(\sigma_{e-1}\right)\right)=s_{e}^{*}\left(p_{e}^{*}\left(\sigma_{e}\right)\right)\right)+F_{x_{e}}$ and, by construction, $t_{e-1}^{*}\left(C_{e-1}\right)=s_{e}^{*}\left(C_{e}\right)+a F_{x_{e}}$. We can then write

$$
\bar{C}_{e-1}=t_{e-1}^{*}\left(p_{e-1}^{*}\left(a \sigma_{e-1}+e a f\right)\right)-a F_{x_{e}}-\sum_{i=1}^{h} \nu_{i} E_{i} .
$$

Finally, the canonical formula on $X_{e}$ gives

$$
K_{X_{e}}=s_{e}^{*}\left(K_{S_{e}}\right)+E_{x_{e}}=t_{e-1}^{*}\left(K_{X_{e-1}}\right)+F_{x_{e}},
$$

so that $\bar{Z}_{e} \in H^{0}\left(X_{e}, s_{e}^{*}\left(-K_{S_{e}}-E_{x_{e}}\right)=H^{0}\left(X_{e},-K_{X_{e}}\right)\right.$ is also the strict transform of a unique divisor

$$
Z_{e-1} \in H^{0}\left(S_{e-1},-K_{S_{e-1}}\right)
$$

whose projection

$$
Z_{0, e-1}:=p_{e-1}\left(Z_{e-1}\right) \subset \mathbb{F}_{e-1}
$$

contains all the singular locus of $C_{0, e-1}$. The following lemma completes the proof of Proposition 11.2(iii).

Lemma 11.8. If $S_{0}=\mathbb{P}(E)$, there exists another minimal model $p^{\prime}: S \rightarrow \mathbb{P}^{2}$.

Proof. We start from the usual maps

$$
p: S \longrightarrow S_{0}, \quad q: S \longrightarrow \bar{S} \subset \mathbb{P}^{g},
$$

Repeatedly applying the previous construction, we get a diagram

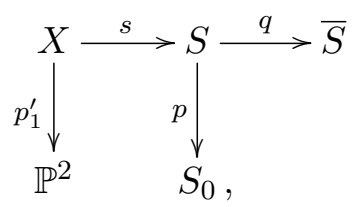

where $s$ is the blow-up of $S$ at points $x_{2}, \ldots, x_{e}$ with exceptional divisors $E_{x_{2}}, \ldots, E_{x_{e}}$ and $p_{1}^{\prime}$ is the composition of the following maps: the blow-up of $\mathbb{P}^{2}$ at a point $P$, the blow-up $\widetilde{\mathbb{F}}_{1}$ of $\mathbb{F}_{1}$ with exceptional divisors $E_{1}, \ldots, E_{h}$ and the blow-up $X$ of $\widetilde{\mathbb{F}}_{1}$ with exceptional divisors $F_{x_{2}}, \ldots, F_{x_{e}}$. The proper transform of $C \subset S$ on $X$ maps via $p_{1}^{\prime}$ onto a plane curve $C_{1}$ whose singular locus is contained in a unique anti-canonical divisor. In order to prove the lemma, we need to show that there exist maps

$$
p_{1}^{\prime}: X \longrightarrow \mathbb{P}^{2}, \quad p_{1}^{\prime \prime}: X \longrightarrow \mathbb{P}^{2},
$$

where $X$ dominates the graph of the birational map $p_{1}^{\prime \prime} \cdot p_{1}^{\prime-1}$, the divisors $E_{x_{2}}, \ldots, E_{x_{e}}$ are $p_{1}^{\prime \prime}$ exceptional and the points $p_{1}^{\prime \prime}\left(E_{x_{i}}\right)$ for $i=2, \ldots, e$ do not lie on the image $C_{0} \subset \mathbb{P}^{2}$ of the proper transform of $C_{1}$. Indeed, if we have such a diagram, the map $p_{1}^{\prime \prime}$ factors through $s$ because the divisors $E_{x_{2}}, \ldots, E_{x_{e}}$ are also $s$-exceptional and we obtain maps

$$
p^{\prime}: S \longrightarrow \mathbb{P}^{2}, \quad q: S \longrightarrow \bar{S}
$$

where $p_{1}^{\prime \prime}=p^{\prime} \cdot s$. In order to construct $p_{1}^{\prime \prime}$, let us consider the plane curve $C_{1}$. The plane curve $C_{1}$ has degree $a e$, it has a point $P$ of multiplicity $a(e-1)$ and $e-1$ points $f_{x_{2}}, \ldots, f_{x_{e}}$ of multiplicity $a$, infinitely near to $P$. Furthermore, it has points $y_{1}, \ldots, y_{h}$, distinct from $P$ and not infinitely near 


\section{ON HYPERPLANE SECTIONS OF K3 SURFACES}

to $P$, with multiplicities $a>\nu_{1}, \ldots, \nu_{h} \geqslant 1$. Note that the proper transform under $p_{1}^{\prime}$ of the lines $l_{x_{i}}$ through $P$ in the directions $f_{x_{i}}$ are the exceptional curves $E_{x_{i}}$ in $X$, and also note that for each $i=2, \ldots, e$, we have $C \cdot E_{x_{i}}=0$. If we perform a first (degenerate) standard quadratic transformation $\Phi_{2}$ of the plane (see [Dol12, Example 7.1.9]) with centres at $P$, at $f_{x_{2}}$ and at $y_{1}$ (notice that $h>0$ ), we obtain a plane curve $C_{1}^{\prime}$ of degree $e a-\nu_{1}$. The curve $C_{1}^{\prime}$ has the same singular points of $C_{1}$ except for the three chosen points, which are replaced by points of multiplicities zero, $(e-1) a-\nu_{1}$ and $a-\nu_{1}$, respectively. The point $x_{2}:=\Phi_{2}\left(E_{x_{2}}\right)$ has, by construction, multiplicity zero and does not to lie on $C_{1}^{\prime}$. The points $f_{x_{3}}, \ldots, f_{x_{e}}$ will be infinitely near the point of multiplicity $(e-1) a-\nu_{1}$, and we can iterate such a construction because we observed that for each $i=2, \ldots, e$, we have $C \cdot E_{x_{i}}=0$. We obtain in such a way the contraction of the divisors $E_{x_{i}}$ with the property that the points $\Phi_{i}\left(E_{x_{i}}\right)$ do not belong to the image $C_{0}$ of the curve $C_{1}$.

This means that the composition $\Phi_{e} \cdots \Phi_{2}$ is the desired birational transformation inducing the sought after map $p_{1}^{\prime \prime}: X \longrightarrow \mathbb{P}^{2}$.

\subsection{The case of $\mathbb{P}^{2}$}

We now consider the case in which the minimal model of $\bar{S}$ is $\mathbb{P}^{2}$. Our aim is to prove Proposition 11.2(iv). As usual, we consider the desingularisation

$$
q: S \longrightarrow \bar{S} \subset \mathbb{P}^{g}
$$

and the natural morphism

$$
p: S \longrightarrow S_{0}=\mathbb{P}^{2}
$$

From Proposition 9.2, there is a unique section $J \in H^{0}\left(S,-K_{S}\right)$ which is contracted to a point by $q$. Set $C_{0}=p(C) \subset \mathbb{P}^{2}$ and $J_{0}=\pi(J) \subset \mathbb{P}^{2}$. The plane curve $J_{0}$ is a cubic passing through the singular points of $C_{0}$. We denote by the same symbol $\ell$ the class of a line in $\mathbb{P}^{2}$ and its pull-back on $S$. We have

$$
\begin{aligned}
C & \sim d \ell-\sum_{i=1}^{h} \nu_{i} E_{i}, \quad \nu_{1} \geqslant \cdots \geqslant \nu_{h} \geqslant 1, \\
K_{S} & \sim-3 \ell+\sum_{i=1}^{h} E_{i} .
\end{aligned}
$$

The condition $J \cdot C=0$ gives

$$
3 d=\sum_{i=1}^{h} \nu_{i} .
$$

Furthermore, since $|C|$ contracts $J$, we have $J^{2}<0$, so that, in particular, $h \geqslant 10$. By Corollary 10.4, we may assume that $J_{0}$ is smooth and that $P_{1}=p\left(E_{1}\right), \ldots, P_{h}=p\left(E_{h}\right)$ are distinct points of $J_{0}$, no three on a line. Assume $\nu_{1}+\nu_{2}+\nu_{3}>d$. The quadratic transformation of $\mathbb{P}^{2}$ centred at $P_{1}, P_{2}, P_{3}$ replaces $C_{0}$ by $\widetilde{C}_{0}$ of degree $\widetilde{d}=2 d-\left(\nu_{1}+\nu_{2}+\nu_{3}\right)<d$ and meeting the smooth cubic $\widetilde{J}_{0}$, the proper transform of $J_{0}$, again at $h$ points $\widetilde{P}_{1}, \ldots, \widetilde{P}_{h}$, of multiplicities say $\widetilde{\nu}_{1} \geqslant \cdots \geqslant \widetilde{\nu}_{h}$. By Corollary 10.4 again, we may assume that $\widetilde{P}_{1}, \ldots, \widetilde{P}_{h}$ are distinct and that no three lie on a line. By repeating this process, we can arrive at a situation where

$$
\nu_{1}+\nu_{2}+\nu_{3} \leqslant d,
$$




\section{E. Arbarello, A. Bruno and E. Sernesi}

and therefore we may assume that this condition holds. From now on, we will set

$$
d=3 n+\epsilon, \quad \epsilon=0,1,2 .
$$

We can then assume

$$
\nu_{3} \leqslant n
$$

We find it convenient to introduce the following notation:

$$
b_{i}=n-\nu_{i}, \quad i=1, \ldots, h .
$$

We can then write the class of $C$ in the following way:

$$
C=-n K_{S}+\epsilon \ell+\sum_{i} b_{i} E_{i}=(3 n+\epsilon) \ell-\sum_{1}^{h}\left(n-b_{i}\right) E_{i} .
$$

The inequalities (11.10) and (11.12) imply

$$
\begin{gathered}
b_{1} \leqslant \cdots \leqslant b_{h} \\
b_{1}+b_{2}+b_{3}+\epsilon \geqslant 0 .
\end{gathered}
$$

In particular, $0 \leqslant b_{3}$. We also have

$$
K_{S} \cdot C=0, \quad C^{2}=d^{2}-\sum \nu_{i}^{2}=(3 n+\epsilon)^{2}-\sum_{i}\left(n-b_{i}\right)^{2},
$$

and therefore

$$
\begin{aligned}
g:=g(C) & =\frac{1}{2}\left(K_{S}+C \cdot C\right)+1=\frac{1}{2} C^{2}+1 \\
& =\frac{1}{2}\left[d^{2}-\sum \nu_{i}^{2}\right]+1 \\
& =\frac{1}{2}\left[(3 n+\epsilon)^{2}-\sum_{i}\left(n-b_{i}\right)^{2}\right]+1 .
\end{aligned}
$$

Now, consider the pencil

$$
|M|:=\left|3 l-\sum_{i=1}^{8} E_{i}\right|=\left|-K_{S}+\sum_{i \geqslant 9} E_{i}\right|
$$

on $S$. We use this pencil as a probe for the Brill-Noether-Petri property. Exactly as in the case of an elliptic ruled surface, we have the sequence (11.4) and the equalities $h^{0}(S, M-C)=0$ and, by the generality of the eight points, $h^{0}(S, M)=2$. Thus $M_{C}:=M \otimes \mathcal{O}_{C}$ is at least a pencil. Enforcing the Brill-Noether-Petri property on this pencil means that we must assume that both (11.5) and (11.6) hold.

Moreover,

$$
\begin{aligned}
\operatorname{deg}\left(M_{C}\right) & =\left(3 \ell-\sum_{i \leqslant 8} E_{i}\right) \cdot\left((3 n+\epsilon) \ell-\sum_{1}^{h}\left(n-b_{i}\right) E_{i}\right) \\
& =n+3 \epsilon+\sum_{i \leqslant 8} b_{i} .
\end{aligned}
$$

For $k \geqslant 0$, we let

$$
A_{k}=C+k K_{S}=(k-n) K_{S}+\epsilon \ell+\sum_{i} b_{i} E_{i}
$$




\section{ON HYPERPLANE SECTIONS OF K3 SURFACES}

Lemma 11.9. The divisor $A_{k}$ is effective for all $k$ with $0 \leqslant k \leqslant \nu_{3}=n-b_{3}$.

Proof. Since $-K_{S}$ is effective, it suffices to prove that $A_{\nu_{3}}$ is effective. We have

$$
\begin{aligned}
A_{\nu_{3}} & =-b_{3} K_{S}+\epsilon \ell+\sum_{i} b_{i} E_{i} \\
& =\left(3 b_{3}+\epsilon\right) \ell-\left(b_{3}-b_{1}\right) E_{1}-\left(b_{3}-b_{2}\right) E_{2}+D,
\end{aligned}
$$

where $D=\sum_{i \geqslant 4}\left(b_{i}-b_{3}\right) E_{i}$ is effective. The inequality (11.15) can be rephrased as

$$
3 b_{3}+\epsilon \geqslant\left(b_{3}-b_{1}\right)+\left(b_{3}-b_{2}\right),
$$

and this implies that $\left(3 b_{3}+\epsilon\right) \ell-\left(b_{3}-b_{1}\right) E_{1}-\left(b_{3}-b_{2}\right) E_{2}$ is effective.

Proposition 11.2(iv) follows immediately from the following corollary, which then concludes the proof of Proposition 11.2.

Corollary 11.10. Suppose that $C$ is BNP and $n \geqslant 2$. Then only the following possibilities may occur:

(i) $\epsilon=0, \nu_{1}=\nu_{2}=\nu_{3}=n, n-1 \leqslant \nu_{9} \leqslant n, d=3 n$;

(ii) $\epsilon=1, \nu_{1}=\nu_{2}=\nu_{3}=n, n-1 \leqslant \nu_{9} \leqslant n, d=3 n+1$;

(iii) $\epsilon=1, \nu_{1}=n+1, \nu_{2}=\nu_{3}=n, n-1 \leqslant \nu_{9} \leqslant n, d=3 n+1$;

(iv) $\epsilon=2, \nu_{1}=\nu_{2}=n+1, \nu_{3}=n, n-1 \leqslant \nu_{9} \leqslant n, d=3 n+2$.

Equivalently, we may have

(i) $\epsilon=0, b_{1}=b_{2}=b_{3}=0,0 \leqslant b_{9} \leqslant 1$,

(ii) $\epsilon=1, b_{1}=b_{2}=b_{3}=0,0 \leqslant b_{9} \leqslant 1$,

(iii) $\epsilon=1, b_{1}=-1, b_{2}=b_{3}=0,0 \leqslant b_{9} \leqslant 1$,

(iv) $\epsilon=2, b_{1}=b_{2}=-1, b_{3}=0,0 \leqslant b_{9} \leqslant 1$.

Furthermore, $h \leqslant 18$.

Proof. Let $N=\ell-E_{1}$ and $N_{C}=N \otimes \mathcal{O}_{C}$. Since $N_{C}$ is at least a pencil and $C$ is a BNP curve, we must have $h^{0}\left(C, K_{C}-2 N_{C}\right)=0$, which implies $h^{0}(S, C-2 N)=0$. We have

$$
\begin{aligned}
C-2 N+\nu_{3} K_{S} & =A_{\nu_{3}}-2 N \\
& =\left(3 b_{3}+\epsilon-2\right) \ell-\left(b_{3}-b_{1}-2\right) E_{1}-\left(b_{3}-b_{2}\right) E_{2}+D,
\end{aligned}
$$

where $D=\sum_{i \geqslant 4}\left(b_{i}-b_{3}\right) E_{i}$ is effective. Since we have

$$
C-2 N=\left(3 b_{3}+\epsilon-2\right) \ell-\left(b_{3}-b_{1}-2\right) E_{1}-\left(b_{3}-b_{2}\right) E_{2}+D-\nu_{3} K_{S}
$$

and $-\nu_{3} K_{S}$ is effective, the divisor $F=\left(3 b_{3}+\epsilon-2\right) \ell-\left(b_{3}-b_{1}-2\right) E_{1}-\left(b_{3}-b_{2}\right) E_{2}$ cannot be effective. But we have, by (11.15),

$$
\left(3 b_{3}+\epsilon-2\right) \geqslant\left(b_{3}-b_{1}-2\right)+\left(b_{3}-b_{2}\right),
$$

and therefore either $3 b_{3}+\epsilon-2<0$ or $b_{3}-b_{1}-2<0$ (otherwise $F$ is effective, arguing as in the proof of Lemma 11.9). But since $b_{3} \geqslant 0$ and $b_{3}-b_{2} \geqslant 0$, the following cases may a priori occur:

- $3 b_{3}+\epsilon-2<0$, and then

$-\epsilon=0, b_{1}=b_{2}=b_{3}=0$,

$-\epsilon=1, b_{1}=b_{2}=b_{3}=0$, 


\section{E. Arbarello, A. Bruno and E. Sernesi}

$-\epsilon=1, b_{1}=-1, b_{2}=b_{3}=0$.

- $3 b_{3}+\epsilon-2=0, b_{3}-b_{1}-2<0$ and $b_{3}-b_{2}>0$, and then

$-\epsilon=2, b_{1}=b_{2}=-1, b_{3}=0$.

The case $3 b_{3}+\epsilon-2>0$ cannot occur: in fact, the other condition $b_{3}-b_{1}-2<0$ implies $b_{3}-b_{1} \leqslant 1$, and therefore also $b_{3}-b_{2} \leqslant 1$, and any such choice implies that $F$ is effective.

Now, consider the pencil $\left|M_{C}\right|$ and impose the Petri condition to it. This means $h^{0}\left(C, K_{C}-\right.$ $\left.2 M_{C}\right)=0$, and since $-2 M$ is ineffective, this implies $h^{0}(S, C-2 M)=0$. On the other hand,

$$
\begin{aligned}
C-2 M+\left(\nu_{3}-2\right) K_{S} & =C+\nu_{3} K_{S}-2 \sum_{i \geqslant 9} E_{i}=A_{\nu_{3}}-2 \sum_{i \geqslant 9} E_{i} \\
& =\epsilon \ell+\sum_{1 \leqslant i \leqslant 8} b_{i} E_{i}+\sum_{j \geqslant 9}\left(b_{j}-2\right) E_{j} \quad\left(\text { since } b_{3}=0\right) .
\end{aligned}
$$

Assume for a contradiction that $b_{9} \geqslant 2$. Then also $b_{j} \geqslant 2$ for all $j \geqslant 9$. This implies that $C-2 M+\left(\nu_{3}-2\right) K_{S}$ is effective. But then

$$
C-2 M=\left[C-2 M+\left(\nu_{3}-2\right) K_{S}\right]+\left(\nu_{3}-2\right)\left(-K_{S}\right)
$$

is effective as well since $\nu_{3}=n \geqslant 2$. Let us finally prove that, under our hypotheses, we have $h \leqslant 18$. A case-by-case inspection using equation (9.3) shows that conditions (i)-(iv) imply $h \leqslant 18$ with only one exception, namely when $\epsilon=2, \nu_{1}=\nu_{2}=n+1, \nu_{3}=n, \nu_{4}=n-1$. In this case $h=19$. In this case, however, from (11.11) and from the genus formula, we get $g=10 n-6$, while $\operatorname{deg}\left(M_{C}\right)=n+9$. Hence, imposing that $\left|M_{C}\right|$ is a Brill-Noether pencil, gives $2 n+18-10 n+4 \geqslant 0$, so that $n=2$. As a consequence, $C$ is a degree 8 plane curve of genus 14 having a triple point. Thus $C$ is not a Brill-Noether curve and this case must be excluded.

\section{ACKNOWLEDGEMENTS}

We would like to thank Claire Voisin for her encouragement at a crucial stage of our project. We also thank the referee for a very careful reading and many useful remarks.

\section{REFERENCES}

AB17 E. Arbarello and A. Bruno, Rank-two vector bundles on polarised Halphen surfaces and the Gauss-Wahl map for du Val curves, J. Éc. polytech. Math. 4 (2017), 257-285; https://doi. org/10.5802/jep. 43.

ABFS16 E. Arbarello, A. Bruno, G. Farkas and G. Saccà, Explicit Brill-Noether-Petri general curves, Comment. Math. Helv. 91 (2016), no. 3, 477-491; https://doi.org/10.4171/CMH/392.

ABS14 E. Arbarello, A. Bruno and E. Sernesi, Mukai's program for curves on a K3 surface, Algebr. Geom. 1 (2014), no. 5, 532-557; https://doi.org/10.14231/AG-2014-023.

AC10 K. Altmann and J. A. Christophersen, Deforming Stanley-Reisner schemes, Math. Ann. 348 (2010), no. 3, 513-537; https://doi.org/10.1007/s00208-010-0490-x.

ACG11 E. Arbarello, M. Cornalba and P. Griffiths, Geometry of Algebraic Curves, Vol. II with a contribution by J.D. Harris, Grundlehren math. Wiss., vol. 268 (Springer-Verlag, Berlin Heidelberg, 2011); https://doi.org/10.1007/978-3-540-69392-5.

ACGH85 E. Arbarello, M. Cornalba, P. Griffiths and J. D. Harris, Geometry of Algebraic Curves, Vol. I, Grundlehren math. Wiss., vol. 267 (Springer-Verlag, New York, 1985); https://doi.org/10. 1007/978-1-4757-5323-3. 


\section{ON HYPERPLANE SECTIONS OF K3 SURFACES}

BEL91 A. Bertram, L. Ein and R. Lazarsfeld, Vanishing theorems, a theorem of Severi, and the equations defining projective varieties, J. Amer. Math. Soc. 4 (1991), no. 3, 587-602; https: //doi.org/10.2307/2939270.

Ber97 A. Bertram, An application of the log version of the Kodaira vanishing theorem to embedded projective varieties, 1997, arXiv:alg-geom/9707001.

BM87 A. Beauville and J.-Y. Mérindol, Sections hyperplanes des surfaces K3, Duke Math. J. 55 (1987), no. 4, 873-878; https://doi.org/10.1215/S0012-7094-87-05541-4.

BPvdV84 W. Barth, C. Peters and A. van de Ven, Compact complex surfaces, Ergeb. Math. Grenzgeb. (3), vol. 4, (Springer-Verlag, Berlin, 1984); https://doi.org/10.1007/978-3-642-96754-2.

BW74 D. M. Burns, Jr. and J. M. Wahl, Local contributions to global deformations of surfaces, Invent. Math. 26 (1974), 67-88; https://doi.org/10.1007/BF01406846.

CHM88 C. Ciliberto, J. Harris and R. Miranda, On the surjectivity of the Wahl map, Duke Math. J. 57 (1988), no. 3, 829-858; https://doi.org/10.1215/S0012-7094-88-05737-7.

CL02 C. Ciliberto and A. F. Lopez, On the number of moduli of extendable canonical curves, Nagoya Math. J. 167 (2002), 101-115; http://projecteuclid.org/euclid.nmj/1114649294.

CU93 F. Cukierman and D. Ulmer, Curves of genus ten on K3 surfaces, Compos. Math. 89 (1993), no. 1, 81-90; http://www. numdam.org/item?id=CM_1993__89_1_81_0.

DH88 S. Diaz and J. Harris, Ideals associated to deformations of singular plane curves, Trans. Amer. Math. Soc. 309 (1988), no. 2, 433-468; https://doi.org/10.2307/2000919.

Dol12 I. V. Dolgachev, Classical Algebraic Geometry. A Modern View (Cambridge Univ. Press, Cambridge, 2012); https://doi.org/10.1017/CB09781139084437.

duV33 P. du Val, On rational surfaces whose prime sections are canonical curves, Proc. London Math. Soc. S2-35 (1933), no. 1, 1-13; https://doi.org/10.1112/plms/s2-35.1.1.

Epe83 D. H. J. Epema, Surfaces with canonical hyperplane sections, Indag. Math. (N. S.) 45 (1983), no. 2, 173-184; https://doi.org/10.1016/1385-7258(83)90054-9.

Epe84_, Surfaces with canonical hyperplane sections, CWI Tract, vol. 1 (Centrum Wisk. Inform., Amsterdam, 1984).

FP05 G. Farkas and M. Popa, Effective divisors on $\overline{\mathscr{M}}_{g}$, curves on K3 surfaces, and the slope conjecture, J. Algebraic Geom. 14 (2005), no. 2, 241-267; https://doi.org/10.1090/ S1056-3911-04-00392-3.

GH80 P. Griffiths and J. Harris, On the variety of special linear systems on a general algebraic curve, Duke Math. J. 47 (1980), no. 1, 233-272; https://doi.org/10.1215/ S0012-7094-80-04717-1.

Gie82 D. Gieseker, Stable curves and special divisors: Petri's conjecture, Invent. Math. 66 (1982), no. 2, 251-275; https://doi.org/10.1007/BF01389394.

GLS07 G.-M. Greuel, C. Lossen and E. Shustin, Introduction to Singularities and Deformations, Monogr. Math. (Springer, Berlin, 2007); https://doi.org/10.1007/3-540-28419-2.

Kar77 U. Karras, Deformations of cusp singularities, Several Complex Variables (Proc. Sympos. Pure Math., Vol. XXX, Part 1, Williamstown, Mass., 1975) (Amer. Math. Soc., Providence, R.I., 1977), 37-44.

KLP12 J.H. Keum, Y. Lee and H. Park, Construction of surfaces of general type from elliptic surfaces via $\mathbb{Q}$-Gorenstein smoothing, Math. Z. 272 (2012), no. 3-4, 1243-1257; https://doi.org/10. 1007/s00209-012-0985-0.

Lau77 H. B. Laufer, On minimally elliptic singularities, Amer. J. Math. 99 (1977), no. 6, 1257-1295; https://doi.org/10.2307/2374025.

Laz86 R. Lazarsfeld, Brill-Noether-Petri without degenerations, J. Differential Geom. 23 (1986), no. 3, 299-307; http://projecteuclid.org/euclid.jdg/1214440116.

Loo81 E. Looijenga, Rational surfaces with an anticanonical cycle, Ann. of Math. 114 (1981), no. 2, 267-322; https://doi.org/10.2307/1971295. 


\section{E. Arbarello, A. Bruno and E. Sernesi}

Ort13 A. Ortega, The Brill-Noether curve and Prym-Tyurin varieties, Math. Ann. 356 (2013), no. 3, 809-817; https://doi.org/10.1007/s00208-012-0870-5.

Pin74 H. C. Pinkham, Deformations of algebraic varieties with $G_{m}$ action, Astérisque 20 (Soc. Math. France, Paris, 1974), 1-131.

Pin77 _ Simple elliptic singularities, Del Pezzo surfaces and Cremona transformations, Several Complex Variables (Proc. Sympos. Pure Math., Vol. XXX, Part 1, Williamstown, Mass., 1975) (Amer. Math. Soc., Providence, R.I., 1977), 69-71.

Sch86 F.-O. Schreyer, Syzygies of canonical curves and special linear series, Math. Ann. 275 (1986), no. 1, 105-137; https://doi.org/10.1007/BF01458587.

Ser06 E. Sernesi, Deformations of Algebraic Schemes, Grundlehren math. Wiss., vol. 334 (SpringerVerlag, Berlin, 2006); https://doi.org/10.1007/978-3-540-30615-3.

Szp79 L. Szpiro, Lectures on Equations Defining Space Curves. notes by N. Mohan Kumar, Tata Inst. Fund. Res. Stud. Math. (Springer-Verlag, Berlin - New York, 1979).

Ume81 Y. Umezu, On normal projective surfaces with trivial dualizing sheaf, Tokyo J. Math. 4 (1981), no. 2, 343-354; https://doi.org/10.3836/tjm/1270215159.

Ver02 P. Vermeire, On the regularity of powers of ideal sheaves, Compos. Math. 131 (2002), no. 2, 161-172; https://doi.org/10.1023/A:1014913511483.

Voi88 C. Voisin, Courbes tétragonales et cohomologie de Koszul, J. reine angew. Math. 387 (1988), 111-121; https://doi.org/10.1515/crll.1988.387.111.

Voi92 Sur l'application de Wahl des courbes satisfaisant la condition de Brill-Noether-Petri, Acta Math. 168 (1992), no. 3-4, 249-272; https://doi.org/10.1007/BF02392980.

Wah81 J. Wahl, Smoothings of normal surface singularities, Topology 20 (1981), no. 3, 219-246; https://doi.org/10.1016/0040-9383(81)90001-X.

Wah87 The Jacobian algebra of a graded Gorenstein singularity, Duke Math. J. 55 (1987), no. 4, 843-871; https://doi.org/10.1215/S0012-7094-87-05540-2.

Wah97 On cohomology of the square of an ideal sheaf, J. Algebraic Geom. 6 (1997), no. 3, $481-511$.

Enrico Arbarello ea@mat.uniroma1.it

Dipartimento di Matematica Guido Castelnuovo, Università di Roma Sapienza, Piazzale

A. Moro 2, 00185 Roma, Italy

Andrea Bruno bruno@mat.uniroma3.it

Dipartimento di Matematica e Fisica, Università Roma Tre, L.go S.L. Murialdo 1, 00146 Roma, Italy

Edoardo Sernesi sernesi@mat.uniroma3.it

Dipartimento di Matematica e Fisica, Università Roma Tre, L.go S.L. Murialdo 1, 00146 Roma, Italy 